\title{
On Some Extensions to GA Package: Hybrid Optimisation, Parallelisation and Islands Evolution
}

by Luca Scrucca

\begin{abstract}
Genetic algorithms are stochastic iterative algorithms in which a population of individuals evolve by emulating the process of biological evolution and natural selection. The R package GA provides a collection of general purpose functions for optimisation using genetic algorithms. This paper describes some enhancements recently introduced in version 3 of the package. In particular, hybrid GAs have been implemented by including the option to perform local searches during the evolution. This allows to combine the power of genetic algorithms with the speed of a local optimiser. Another major improvement is the provision of facilities for parallel computing. Parallelisation has been implemented using both the master-slave approach and the islands evolution model. Several examples of usage are presented, with both real-world data examples and benchmark functions, showing that often high-quality solutions can be obtained more efficiently.
\end{abstract}

\section{Introduction}

Optimisation problems of both practical and theoretical importance deal with the search of an optimal configuration for a set of variables to achieve some specified goals. Potential solutions may be encoded with real-valued, discrete, binary or permutation decision variables depending on the problem to be solved. Direct search or derivative-free methods, gradient-based and Newton-type methods, all encompass traditional local optimisation algorithms for real-valued functions (Chong and Zak, 2013; Givens and Hoeting, 2013, Chap. 2). In contrast, discrete and combinatorial optimisation problems involve decision variables expressed using integers or binary values and consist in searching for the best solution from a set of discrete elements (Papadimitriou and Steiglitz, 1998; Givens and Hoeting, 2013, Chap. 3).

A large number of heuristics and metaheuristics algorithms have been proposed for solving complex optimisation tasks. Specific (ad-hoc) heuristic techniques are able to identify solutions in a reasonably short amount of time, but the solutions obtained are generally not guaranteed to be optimal or accurate. On the contrary, metaheuristics offer a tradeoff between exact and heuristics methods, in the sense that they are generic techniques that offer good solutions, often the global optimum value sought, in a moderate execution time by efficiently and effectively exploring the search space (Luke, 2013). This class of algorithms typically implements some form of stochastic optimisation and includes: Evolutionary Algorithm (EA; Back et al., 2000a,b), Iterated Local Search (ILS; Lourenço et al., 2003), Simulated Annealing (SA; Kirkpatrick et al., 1983), Differential Evolution (DE; Storn and Price, 1997), Particle Swarm Optimisation (PSO; Kennedy and Eberhart, 1995), Tabu Search (TS; Glover and Laguna, 2013), Ant Colony Optimisation (ACO; Dorigo and Stützle, 2004), and Covariance Matrix Adaptation Evolution Strategy (CMA-ES; Hansen, 2006).

EAs are stochastic iterative algorithms in which a population of individuals evolve by emulating natural selection (Eiben and Smith, 2003; De Jong, 2006; Simon, 2013). Each individual of the population represents a tentative solution to the problem. The quality of the proposed solution is expressed by the value of a fitness function assigned to each individual. This value is then used by EAs to guide the search and improve the fitness of the population. Compared to other metaheuristics algorithms, EAs are able to balance between exploration of new areas of the search space and exploitation of good solutions. The trade-off between exploration and exploitation is controlled by some tuning parameters, such as the population size, the genetics operators (i.e. selection, crossover, and mutation), and the probability of applying them. Genetic Algorithms (GAs) are search and optimisation procedures that are motivated by the principles of natural genetics and natural selection. GAs are the "earliest, most well-known, and most widely-used EAs" (Simon, 2013, p. 35).

$\mathrm{R}$ offers several tools for solving optimisation problems. A comprehensive listing of available packages is contained in the CRAN task view on "Optimization and Mathematical Programming" (Theussl and Borchers, 2015). An extensive treatment of optimisation techniques applied to problems that arise in statistics and how to solve them using $\mathrm{R}$ is provided by Nash (2014). A gentle introduction to metaheuristics optimisation methods in R is contained in Cortez (2014). Some R packages implementing evolutionary optimisation algorithms are: rgenoud, Rmalschains, DEoptim, GenSA, pso, cmaes, tabuSearch.

The R package GA is a flexible general-purpose set of tools for optimisation using genetic algo- 
rithms and it is fully described in Scrucca (2013). Real-valued, integer, binary and permutation GAs are implemented, whether constrained or not. Discrete or combinatorial optimisation problems, where the search space is made of a finite or countably infinite set of potential solutions, can be easily treated by adopting a binary or permutation representation. Several genetic operators for selection, crossover, and mutation are available, and more can be defined by experienced $\mathrm{R}$ users.

This paper describes some recent additions to the GA package. The first improvement involves the option to use hybrid GAs. Although for many objective functions GAs are able to work in an efficient way and find the area of the global optimum, they are not especially fast at finding the optimum when in a locally quadratic region. Hybrid GAs combine the power of GAs with the speed of a local optimiser, allowing researchers to find a global solution more efficiently than with the conventional evolutionary algorithms. Because GAs can be easily and conveniently executed in parallel machines, the second area of improvement is that associated with parallel computing. Two approaches, the master-slave and islands models, have been implemented and are fully described. Several examples, using both real-world data examples and benchmark functions, are presented and discussed.

\section{GA package}

In the following we assume that the reader has already installed the latest version $(\geq 3.0)$ of the package from CRAN with

> install.packages("GA")

and the package is loaded into an $\mathrm{R}$ session using the usual command

$>$ library(GA)

\section{Hybrid genetic algorithms}

EAs are very good at identifying near-optimal regions of the search space (exploration), but they can take a relatively long time to locate the exact local optimum in the region of interest (exploitation). Traditionally, exploitation is done through selection, whilst exploration is performed by search operators, such as mutation and crossover (Eiben and Schippers, 1998). However, exploitation can also be pursued by controlling crossover and mutation, e.g. by reducing the mutation probability as the search progresses. Balancing between exploration and exploitation is vital for successful application of EAs (Črepinšek et al., 2013).

A further possibility for improving exploitation is to try to incorporate efficient local search algorithms into EAs. There are different ways in which local searches or problem-specific information can be integrated in EAs (see Eiben and Smith, 2003, Chap. 10). For instance, a local search may be started from the best solution found by a GA after a certain number of iterations, so that, once a promising region is identified, the convergence to the global optimum can be sped up. These evolutionary methods have been named in various ways, such as hybrid GAs, memetic GAs, and genetic local search algorithms. Some have argued that the inclusion of a local search in GAs implies the use of a form of Lamarckian evolution. This fact has been criticised from a biological point of view, but "despite the theoretical objections, hybrid genetic algorithms typically do well at optimization tasks" (Whitley, 1994, p. 82).

In case of real-valued optimisation problems, the GA package provides a simple to use implementation of hybrid GAs by setting the argument optim = TRUE in a ga() function call. This allows to perform local searches using the base R function optim(), which makes available general-purpose optimisation methods, such as Nelder-Mead, quasi-Newton with and without box constraints, and conjugate-gradient algorithms.

Having set optim = TRUE, the local search method to be used and other parameters can be controlled with the optional argument optimArgs. This must be a list with the following structure and defaults:

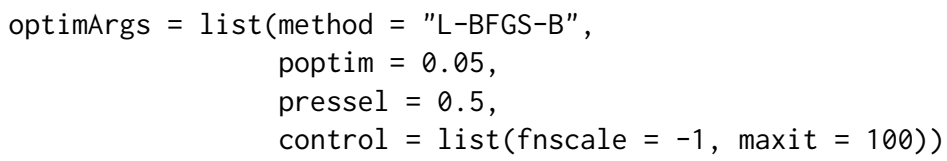

where 
method The method to be used among those available in optim function. By default, the BFGS algorithm with box constraints is used, where the bounds are those provided in the ga() function call. Further methods are available as described in the Details section in help(optim).

poptim A value in the range $(0,1)$ which gives the the probability of applying the local search at each iteration.

pressel A value in the range $(0,1)$ which specifies the pressure selection.

control A list of parameters for fine tuning the optim algorithm. See help (optim) for details.

In the implementation available in GA, the local search is applied stochastically during the GA iterations with probability poptim $\in[0,1]$; by default, once every $1 / 0.05=20$ iterations on average. The local search algorithm is started from a random selected solution drawn with probability proportional to fitness and with the selection process controlled by the parameter pressel $\in[0,1]$. Let $f_{i}$ be the fitness value associated with the $i$ th solution for $i=1, \ldots, n$, where $n$ is the popsize, and let $r_{i}$ be the corresponding rank in non increasing order. Then, for a given pressel the probability of selection is computed as $p_{i}=\operatorname{pressel} \times(1-\text { pressel })^{r_{i}-1}$, and then normalised as $p_{i}=p_{i} / \sum_{i=1}^{n} p_{i}$. Figure 1 shows the probability of selection as a function of the fitness value for different levels of selection pressure. The values on the $y$-axis are computed using the function optimProbsel(), which is used in the GA package for computing the probability of selection for each individual of the genetic population. When the pressure is set at 0 , the same probability of selection is assigned to all solutions. Larger probabilities are assigned to larger $f_{i}$ values as the pressure value increases. In the extreme case of pressure selection equal to 1 , only the largest $f_{i}$ receives a probability of selection equal to 1 , whereas the others have no chance of being selected. Thus, smaller values of pressel tend to assign equal probabilities to all the solutions, and larger values tend to assign larger values to those solutions having better fitness.

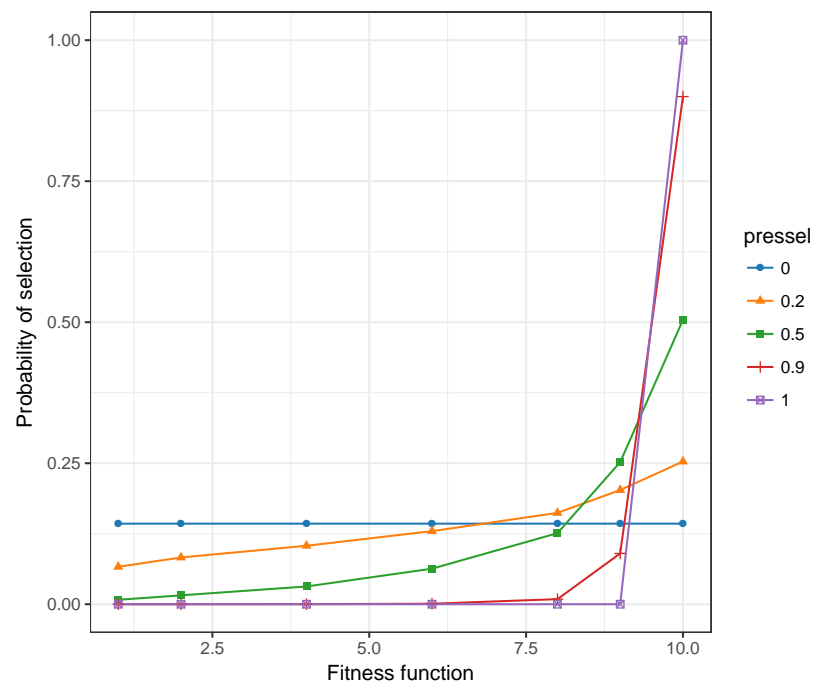

Figure 1: Graph of the probability of selecting a solutions for starting a local search in HGA as a function of the fitness and for different levels of selection pressure.

Note that when a ga() function call is issued with optim = TRUE, a local search is always applied at the end of GA evolution, i.e. after the last iteration and even in case of poptim $=0$, but starting from the solution with the highest fitness value. The rationale for this is to allow for local optimisation as a final improvement step.

\section{Portfolio selection}

In portfolio selection the goal is to find the optimal portfolio, i.e. the portfolio that provides the highest return and lowest risk. This is achieved by choosing the optimal set of proportions of various financial assets (Ruppert and Matteson, 2015, Chap. 16). In this section an example of mean-variance efficient portfolio selection (Gilli et al., 2011, Chap. 13) is illustrated.

Suppose we have selected 10 stocks from which to build a portfolio. We want to determine how 
much of each stock to include in our portfolio. The expected return rate of our portfolio is

$$
E(R)=\sum_{i=1}^{10} w_{i} E\left(R_{i}\right),
$$

where $E\left(R_{i}\right)$ is the expected return rate on asset $i$, and $w_{i}$ is the fraction of the portfolio value due to asset $i$. Note that the portfolio weights $w_{i}$ must satisfy the constraints $w_{i} \geq 0$, and $\sum_{i=1}^{10} w_{i}=1$. At the same time, we want to minimise the variance of portfolio returns given by

$$
\sigma_{p}^{2}=w^{\prime} \Sigma w
$$

where $\Sigma$ is the covariance matrix of stocks returns, and $w^{\prime}=\left(w_{1}, \ldots, w_{10}\right)$, under the constraint that the portfolio must have a minimum expected return of $1 \%$, i.e $E(R) \geq 0.01$. Provided that only linear constraints are included, the problem of mean-variance portfolio selection is typically solved by quadratic programming. However, GAs provide a general approach to portfolio selection that can be used for problems with both linear and nonlinear constraints (Gilli and Schumann, 2012).

Consider the following stocks with monthly return rates obtained by Yahoo finance using the quantmod package:

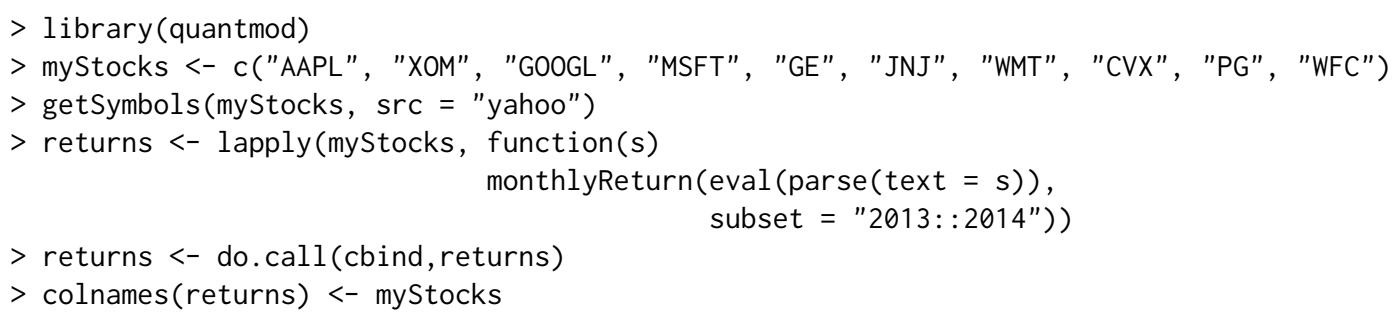

The monthly return rates for the portfolio stocks are shown in Figure 2 and obtained with the code:

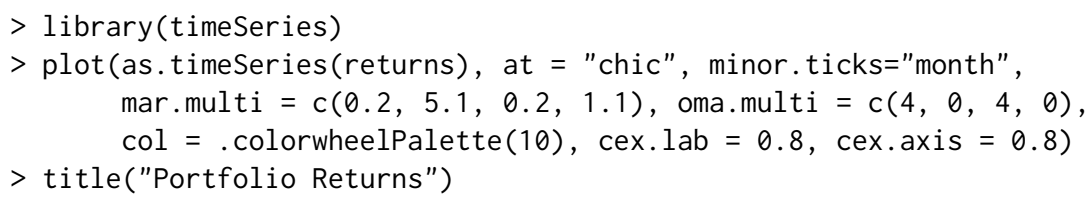

Summary statistics for the portfolio stocks are computed as:

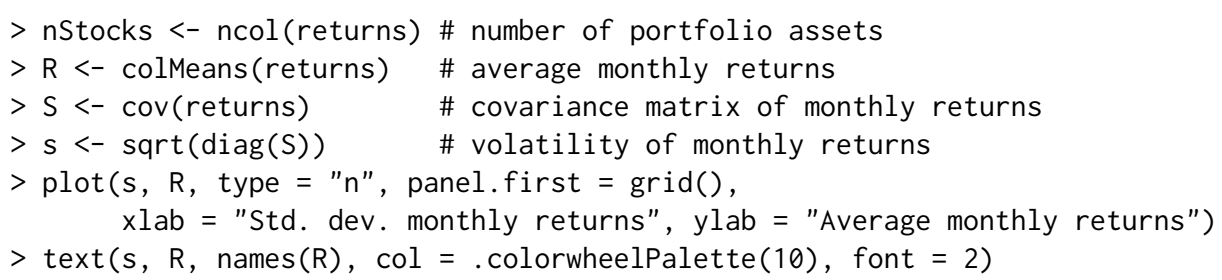

The last two commands draw a graph of the average vs standard deviation for the monthly returns (see Figure 3a). From this graph we can see that there exists a high degree of heterogenity among stocks, with AAPL having the largest standard deviation and negative average return, whereas some stocks have small volatility and high returns, such as WFC and MSFT. Clearly, the latter are good candidate for inclusion in the portfolio. The exact amount of each stock also depends on the correlation among stocks through the variance of portfolio returns $\sigma_{p}^{2}$, and so we need to formalise our objective function under the given constraints.

In order to compute the GA fitness function, we define the following functions:

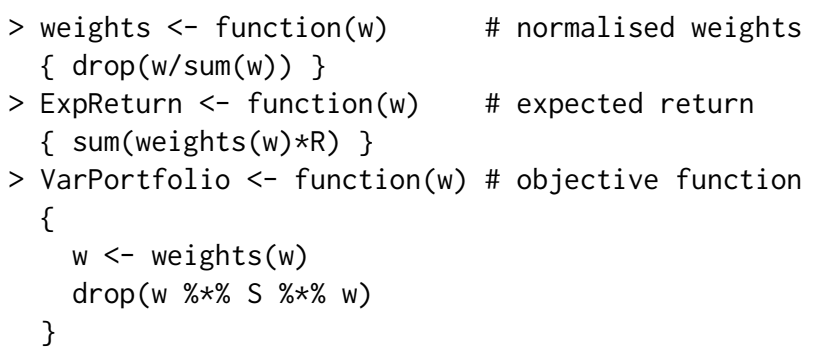

We may define the fitness function to be maximised as the (negative) variance of the portfolio penalised by an amount which is function of the distance between the expected return of the portfolio and the target value: 

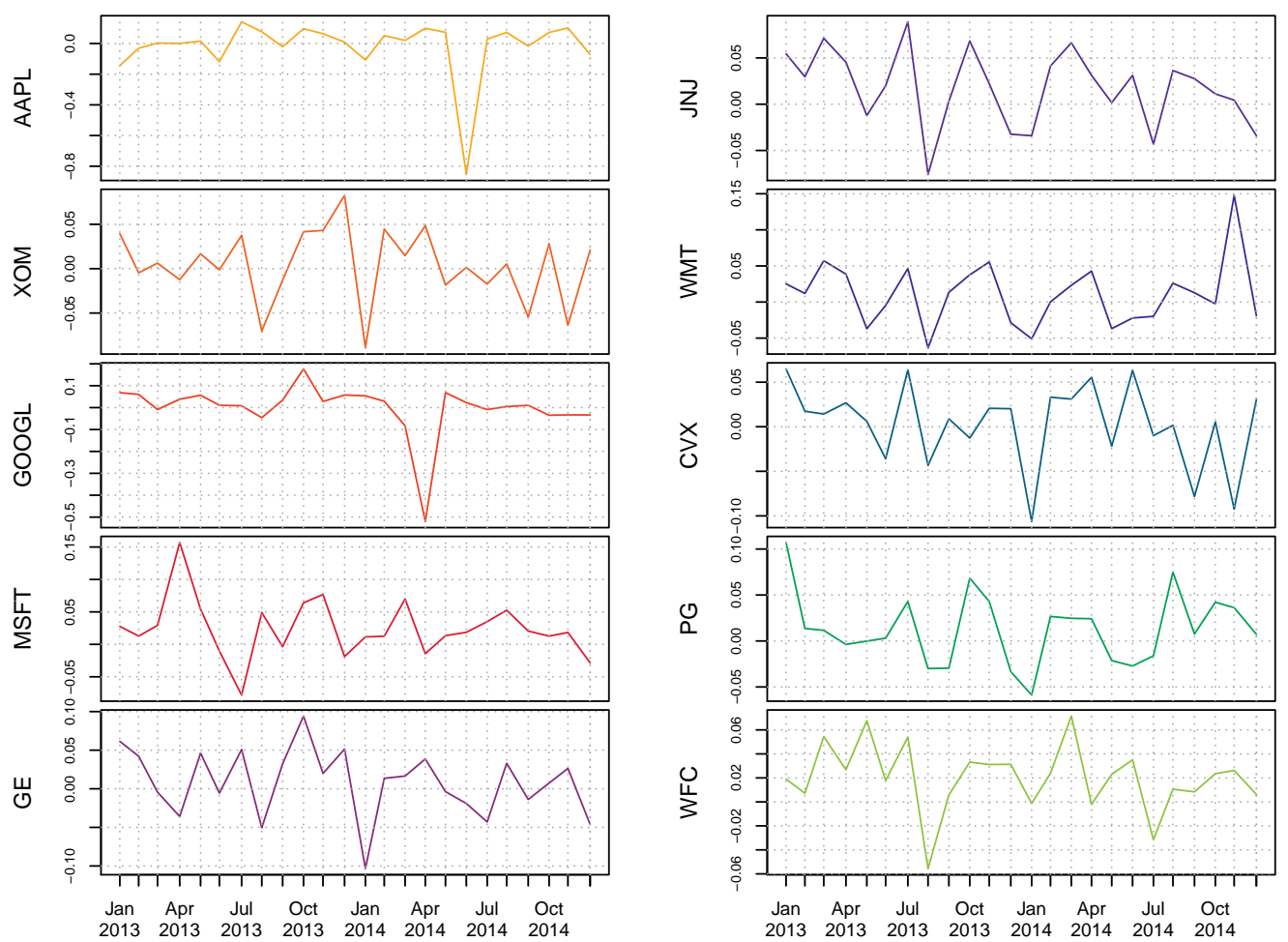

Figure 2: Monthly return rates for a portfolio of selected stocks.

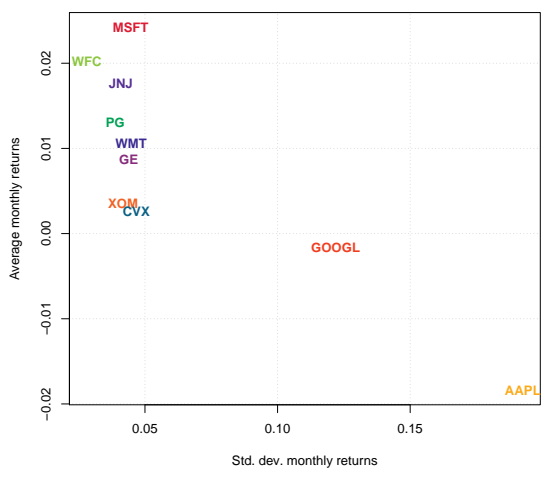

(a)

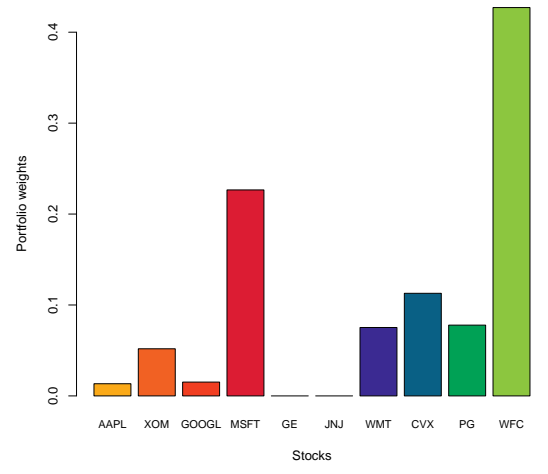

(b)

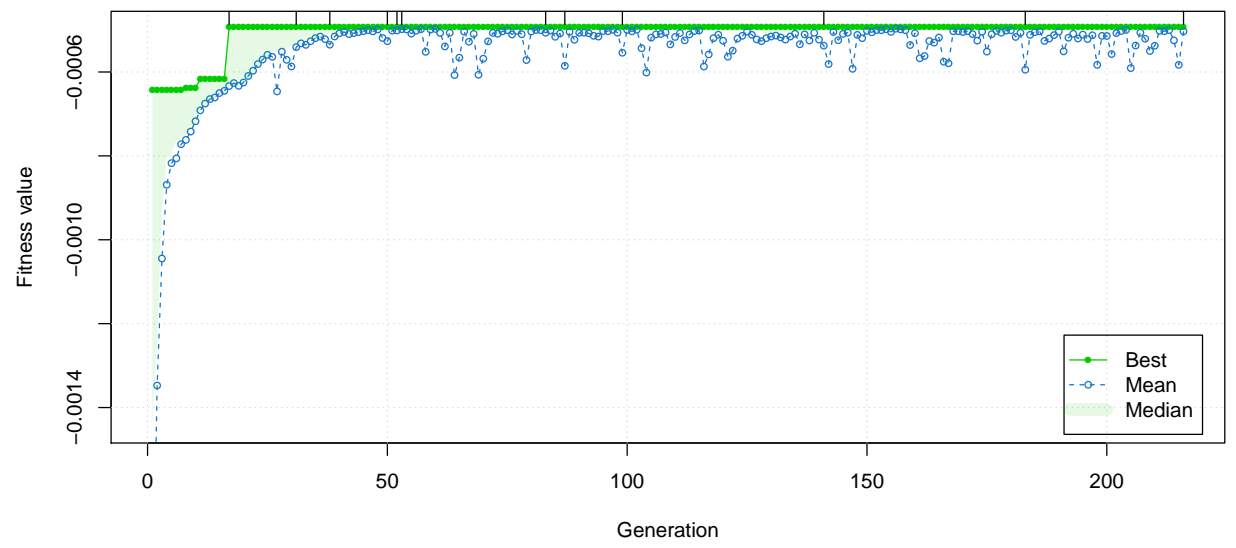

(c)

Figure 3: (a) Plot of average monthly returns vs the standard deviation for the selected stocks. (b) Portfolio stocks composition estimated by HGA. (c) Trace of HGA iterations. 


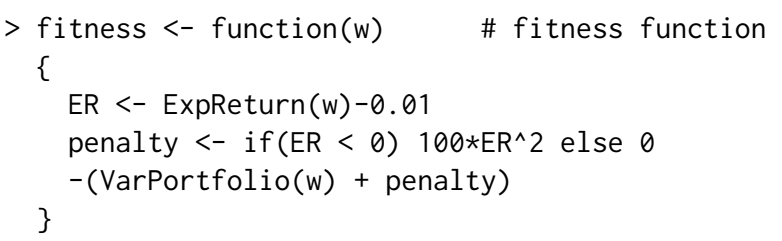

A hybrid GA with local search can be obtained with the following call:

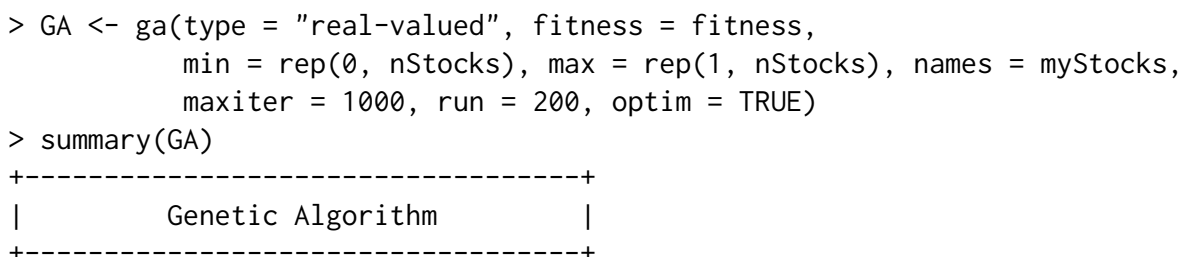

GA settings:

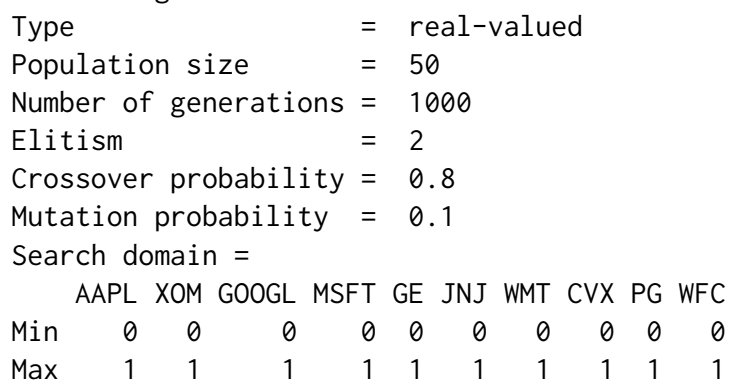

GA results:

Iterations $\quad=216$

Fitness function value $=-0.00049345$

Solution =

AAPL XOM GOOGL MSFT GE JNJ WMT CVX PG WFC

$\begin{array}{lllllllllll}{[1,]} & 0.030918 & 0.11534 & 0.034683 & 0.52062 & 0 & 0 & 0.17201 & 0.26144 & 0.18096 & 0.98719\end{array}$

$>\operatorname{plot}(\mathrm{GA})$

The last command produces the graph on Figure $3 \mathrm{c}$, which shows the trace of best, mean, and median values during the HGA iterations. We also added some vertical dashes at the top of the graph to indicate when the local search occurred. It is interesting to note that the inclusion of a local search greatly speeds up the termination of the GA search, which converges after 216 iterations. Without including the local optimisation step, a fitness function value within a $1 \%$ from the maximum value found above is attained after 1,633 iterations, whereas the same maximum fitness value cannot be achieved even after 100,000 iterations.

The estimated portfolio weights and the corresponding expected return and variance are computed as:

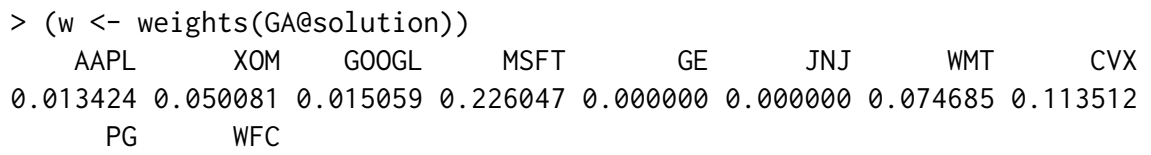

The last command draws a barchart of the optimal portfolio selected, and it is shown in Figure $3 \mathrm{~b}$.

\section{Poisson change-point model}

In the study of stochastic processes a common problem is to determine whether or not the functioning of a process has been modified over time. Change-point models assume that such a change is occurring at some point in time in a relatively abrupt manner (Lindsey, 2004). 
In a single change-point model the distribution of a response variable $Y_{t}$ at time $t$ is altered at the unknown point in time $\tau$, so we can write

$$
Y_{t} \sim \begin{cases}f\left(y_{t} ; \theta_{1}\right) & t<\tau \\ f\left(y_{t} ; \theta_{2}\right) & t \geq \tau\end{cases}
$$

where $f(\cdot)$ is some given parametric distribution depending on $\theta_{k}$ for $k=\{1,2\}$, and $\tau$ is an unknown parameter giving the change-point time. Some or all of the elements of the vector of parameters $\theta_{k}$ in model (1) may change over time. In more complex settings, the distribution function itself may be different before and after the change point.

Given a sample $\left\{y_{t} ; t=1, \ldots, T\right\}$ of observations over time, the log-likelihood function of the change-point problem is

$$
\ell\left(\theta_{1}, \theta_{2}, \tau ; y_{1}, \ldots, y_{T}\right)=\sum_{t<\tau} \log f\left(y_{t} ; \theta_{1}\right)+\sum_{t \geq \tau} \log f\left(y_{t} ; \theta_{2}\right)
$$

Further, for a Poisson change-point model we assume that $f\left(y_{t} ; \theta_{k}\right)$ is the Poisson distribution with mean parameter $\theta_{k}$. Maximisation of (2) can also be seen as a discrete optimisation problem.

Consider the British coal-mining disasters dataset which provides the annual counts of disasters (having at least 10 deaths) from 1851 to 1962 (Jarrett, 1979; Raftery and Akman, 1986). The data from Table 1 of Carlin et al. (1992) are the following:

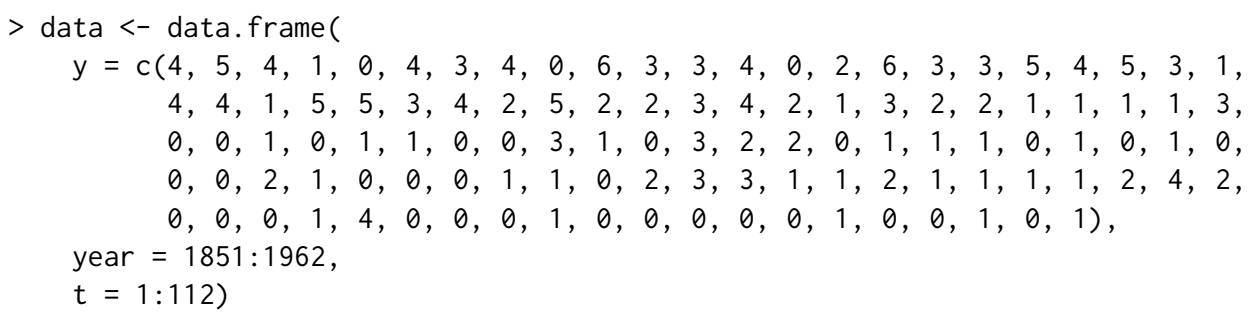

Graphs of annual counts and cumulative sums over time are shown in Figure 4. These can be obtained using the following code:

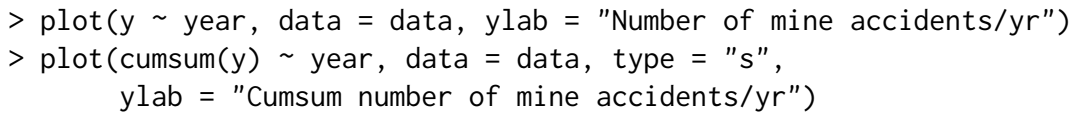

Both graphs seem to suggest a two-regime behaviour for the number of coal-mining disasters.

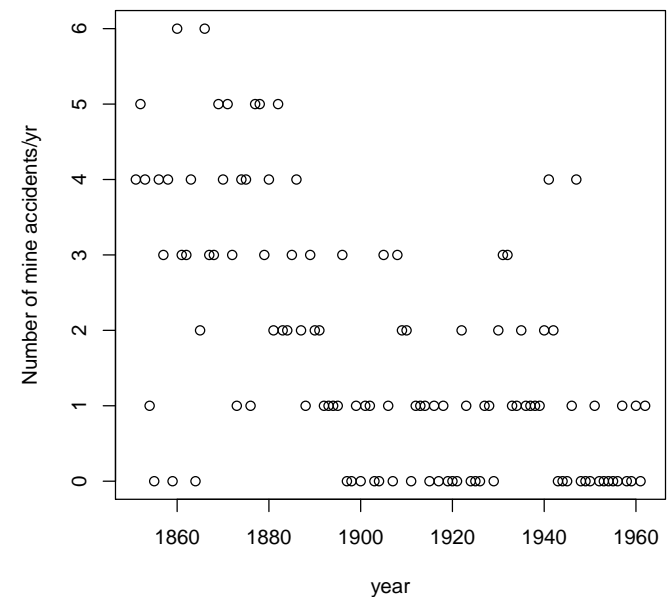

(a)

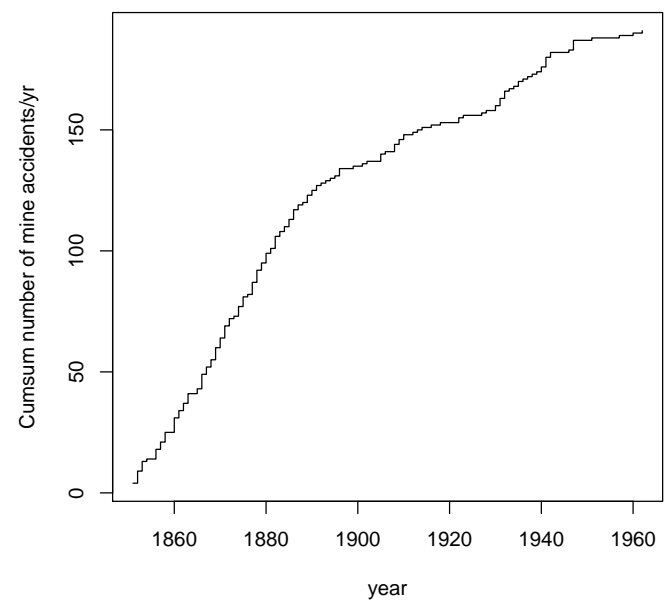

(b)

Figure 4: Plots of the number of yearly coal-mining accidents (a) and cumulative sum of mine accidents (b) from 1851 to 1962 in Great Britain.

We start the analysis by fitting a no change-point model, i.e. assuming a homogeneous Poisson process with constant mean. Clearly, in this simple case the MLE of the Poisson parameter is the sample mean of counts. However, for illustrative purposes we write down the log-likelihood and we maximise it with a hybrid GA. 


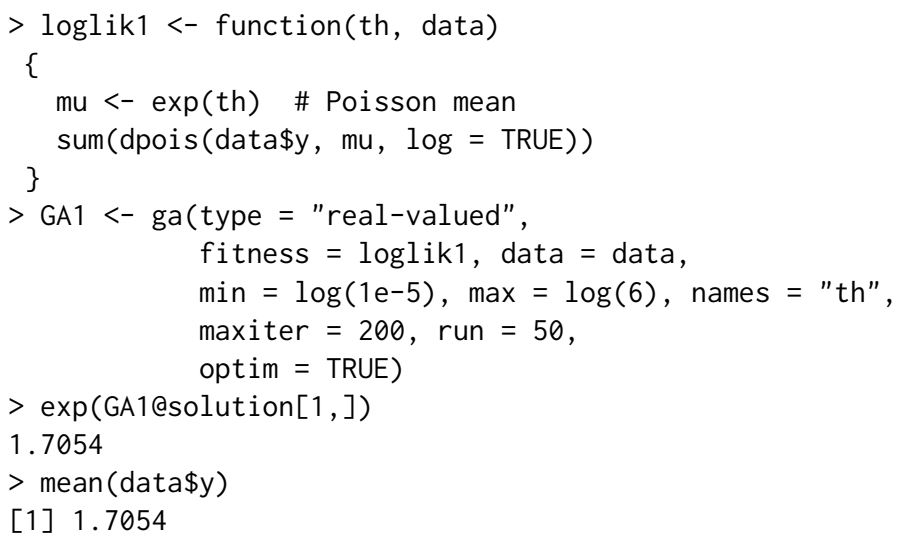

For the change-point model in (1), the mean function can be expressed as

$$
\mu_{t}=\exp \left\{\theta_{1}+\left(\theta_{2}-\theta_{1}\right) I(t \geq \tau)\right\}
$$

where $\tau$ is the time of change-point, $\theta_{1}$ is the mean of the first regime, i.e. when $t<\tau, \theta_{2}$ is the mean of the second regime, i.e. when $t \geq \tau$, and $I(\cdot)$ denotes the indicator function (which is equal to 1 if its argument is true and 0 otherwise). In $\mathrm{R}$ the above mean function and the log-likelihood from (2) can be written as

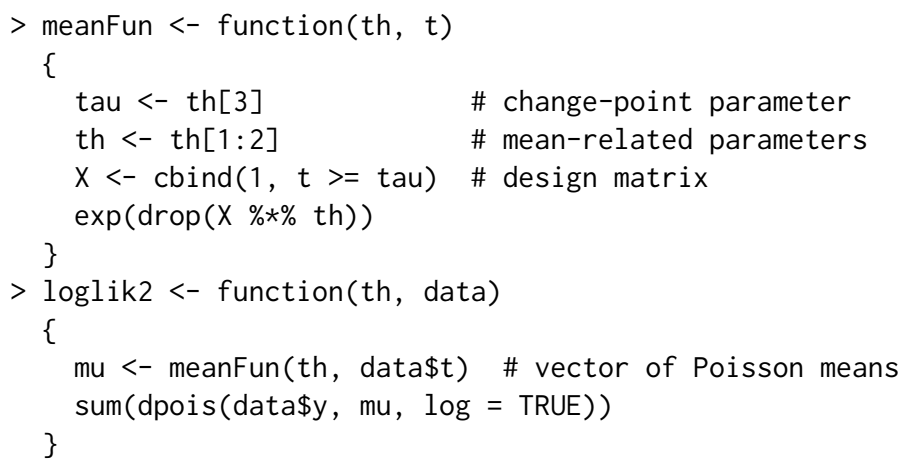

The vector th contains the three parameters that have to be estimated from the sample dataset data. Note that, for convenience, it is defined as $\left(\theta_{1}, \theta_{2}^{*}, \tau\right)^{\prime}$, where $\theta_{2}^{*}=\left(\theta_{2}-\theta_{1}\right)$ is the differential mean effect of second regime.

Direct maximisation of the log-likelihood in loglik2() by iterative derivative-based methods is not viable due to lack of differentiability with respect to $\tau$. However, hybrid GAs can be efficiently used in this case as follows:

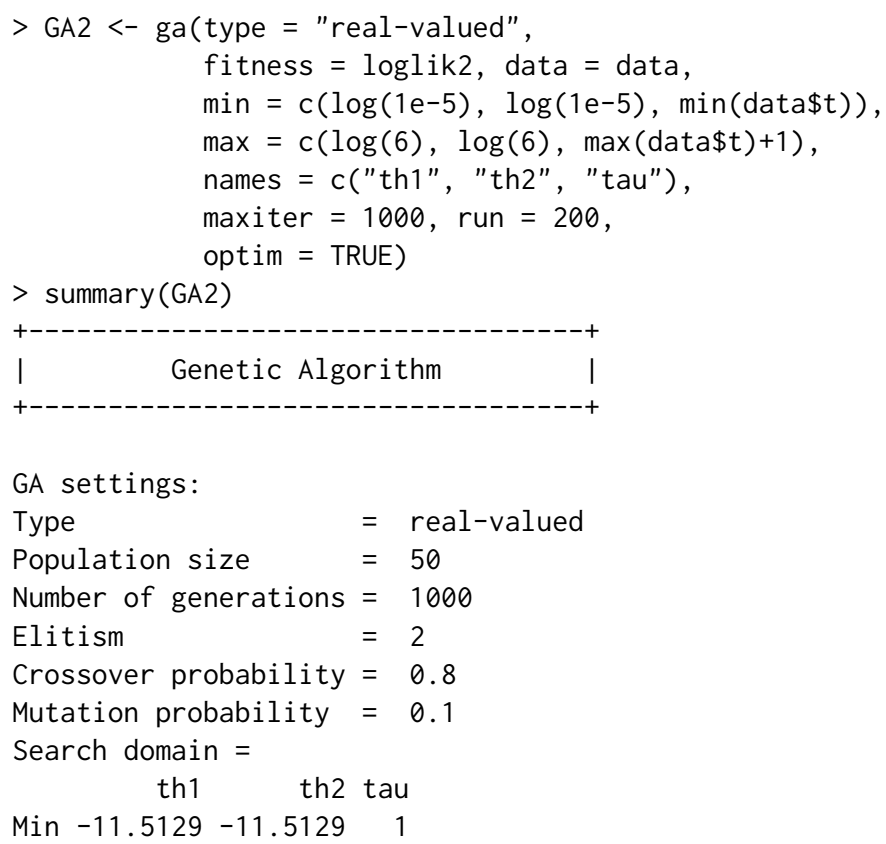




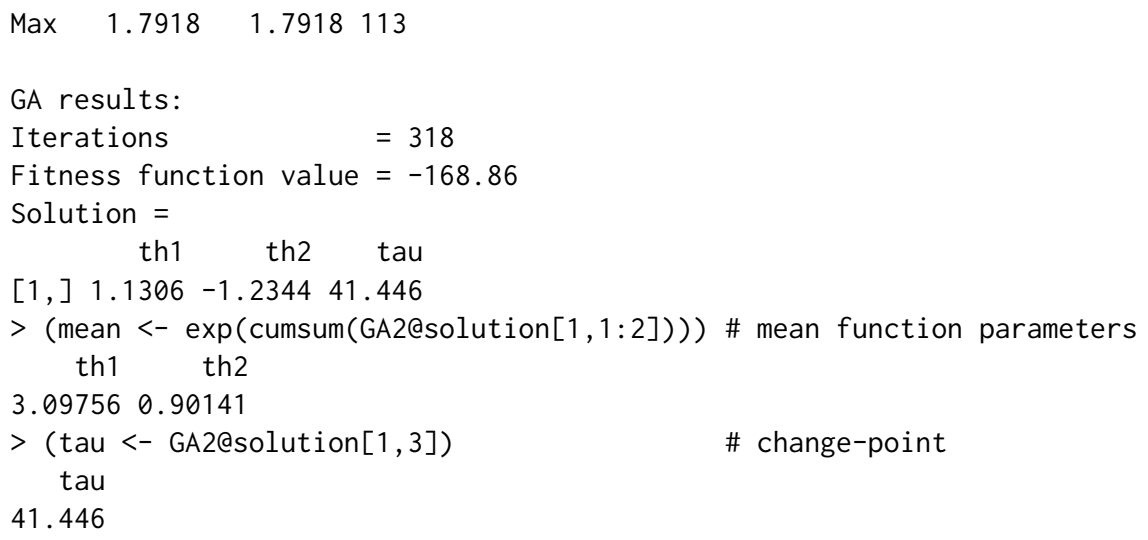

Note that both the estimated change-point and the means are quite close to those reported by Raftery and Akman (1986), and Carlin et al. (1992), using Bayesian methodology.

The two estimated models can be compared using a model selection criterion, such as the Bayesian information criterion (BIC; Schwartz, 1978), defined as

$$
\mathrm{BIC}=2 \ell(\widehat{\theta} ; y)-v \log (n)
$$

where $\ell(\widehat{\theta} ; y)$ is the log-likelihood evaluated at the MLE $\widehat{\theta}, n$ is the number of observations, and $v$ is the number of estimated parameters. Using this definition, larger values of BIC are preferable.

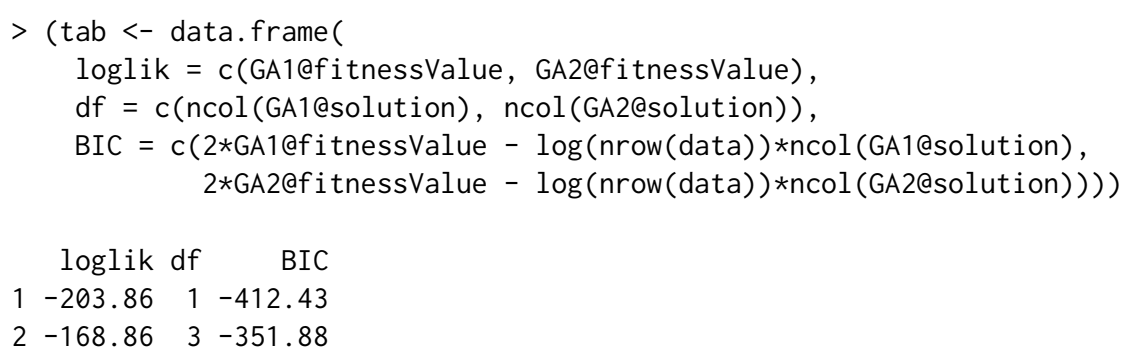

A comparison of BIC values clearly indicates a preference for the change-point model. We may summarise the estimated model by drawing a graph of observed counts over time with the estimated means before and after the change-point:

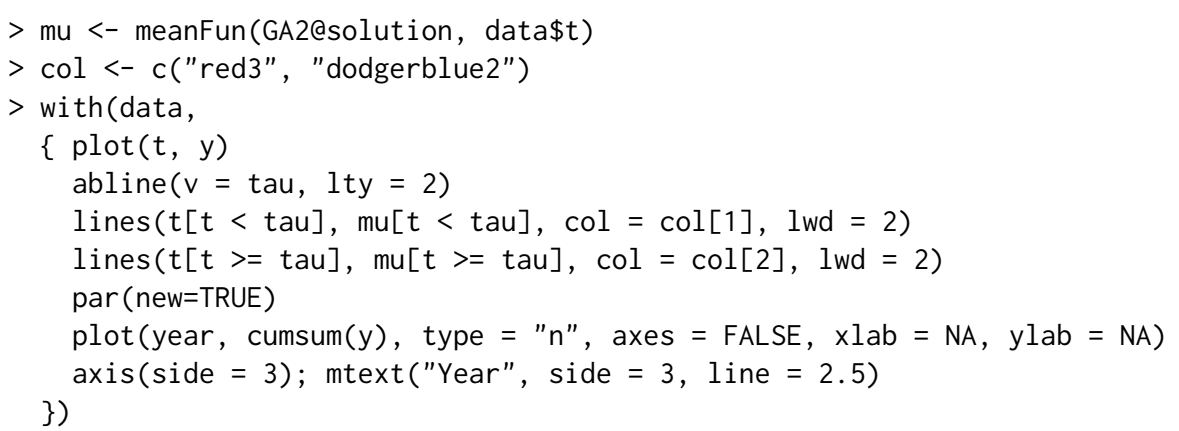

and a graph of observed cumulative counts and the estimated cumulative mean counts:

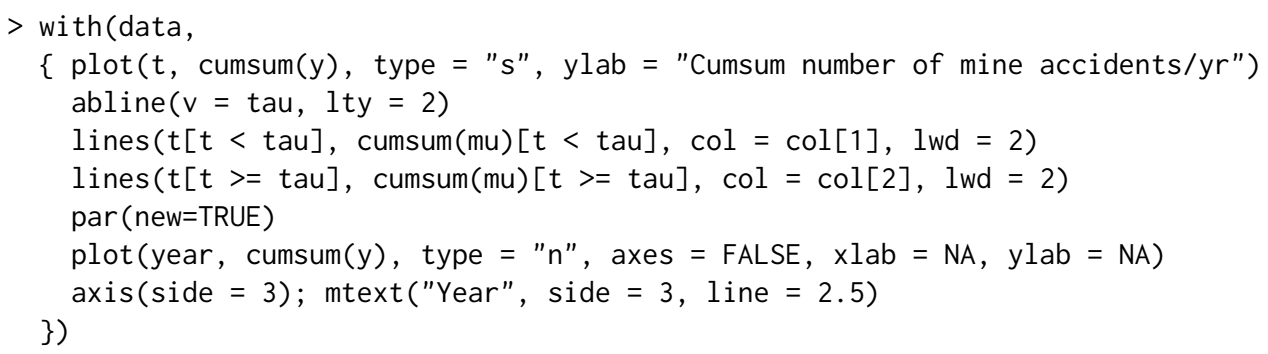

Both graphs are reported in Figure 5. The latter plot is particularly illuminating of the good fit achieved by the selected model. 


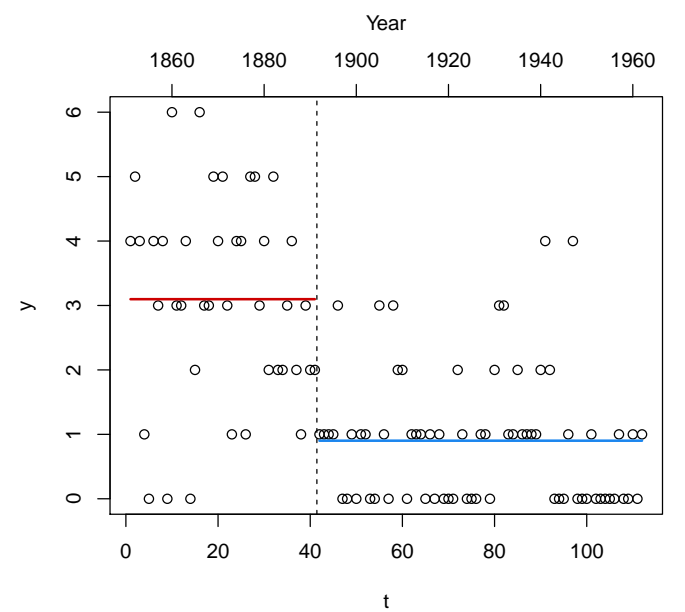

(a)

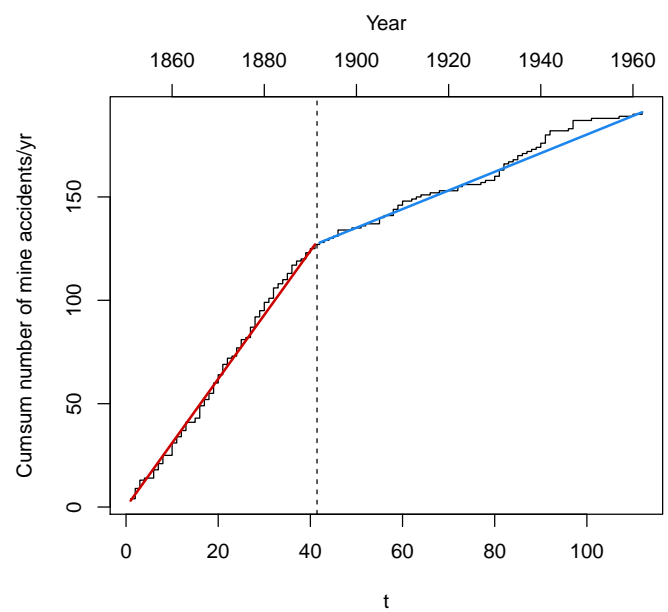

(b)

Figure 5: Summary plots for the change-point model fitted to the British coal-mining accidents dataset: (a) plot of observed counts over time with the estimated means before and after the estimated changepoint (vertical dashed line); (b) plot of observed cumulative counts (step function) and the cumulative estimated mean counts.

\section{Parallel genetic algorithms}

Parallel computing in its essence involves the simultaneous use of multiple computing resources to solve a computational problem. This is viable when a task can be divided into several parts that can be solved simultaneously and independently, either on a single multi-core processors machine or on a cluster of multiple computers.

Support for parallel computing in $\mathrm{R}$ is available since 2011 (version 2.14.0) through the base package parallel. This provides parallel facilities previously contained in packages multicore and snow. Several approaches to parallel computing are available in R (McCallum and Weston, 2011), and an extensive and updated list of $\mathrm{R}$ packages is reported in the CRAN Task View on High-Performance and Parallel Computing with R (Eddelbuettel, 2016, HighPerformanceComputing).

GAs are regarded as "embarrassingly parallel" problems, meaning that they require a large number of independent calculations with negligible synchronisation and communication costs. Thus, GAs are particularly suitable for parallel computing, and it is not surprising that such idea has been often exploited to speed up computations (see for instance Whitley (1994) in the statistical literature).

Luque and Alba (2011) identify several types of parallel GAs. In the master-slaves approach there is a single population, as in sequential GAs, but the evaluation of fitness is distributed among several processors (slaves). The master process is responsible for the distribution of the fitness function evaluation tasks performed by the slaves, and for applying genetic operators such as selection, crossover, and mutation (see Figure 6). Since the latter operations involve the entire population, it is also known as global parallel GAs (GPGA). This approach is generally efficient when the computational time involving the evaluation of the fitness function is more expensive than the communication overhead between processors.

Another approach is the case of distributed multiple-population GAs, where the population is partitioned into several subpopulations and assigned to separated islands. Independent GAs are executed in each island, and only occasionally sparse exchanges of individuals are performed among these islands (see Figure 7). This process, called migration, introduces some diversity into the subpopulations, thus preventing the search from getting stuck in local optima. In principle islands can evolve sequentially, but increased computational efficiency is obtained by running GAs in each island in parallel. This approach is known as coarse-grained GAs or island parallel GAs (ISLPGA).

By default, searches performed with the GA package occur sequentially. In some cases, particularly when the evaluation of the fitness function is time consuming, parallelisation of the search algorithm may be able to speed up computing time. Starting with version 2.0, the GA package provides facilities for using parallel computing in genetic algorithms following the GPGA approach. Recently, with version 3.0, the ISLPGA model has also been implemented in the GA package. The following subsections describes usage of both approaches.

Parallel computing in the GA package requires the following packages to be installed: parallel (available in base R), doParallel, foreach, and iterators. Moreover, doRNG is needed for reproducibil- 


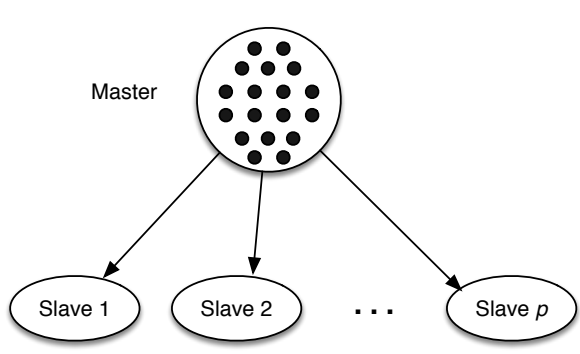

Figure 6: Master-slaves or global parallel GA scheme (GPGA). The master process stores the population, executes genetic operations, and distributes individuals to the slaves, which only evaluate the fitness of individuals.

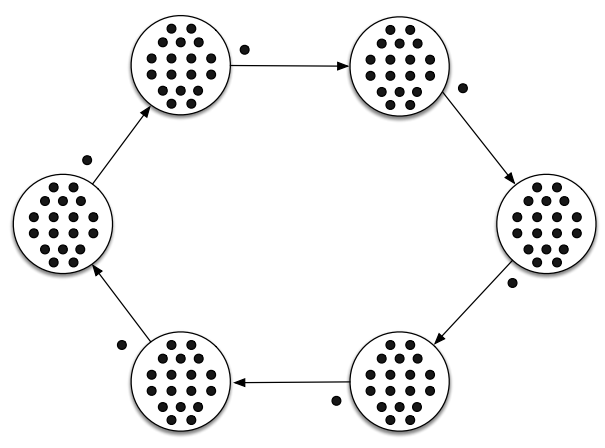

Figure 7: Islands parallel GA scheme (ISLPGA). In a multiple-population parallel GA each process is a simple GA which evolves independently. Individuals occasionally migrate between one island and its neighbours.

ity of results.

\section{Global parallel implementation}

The GPGA approach to parallel computing in GA can be easily obtained by manipulating the optional argument parallel in the ga() function call. This argument accepts several different values. A logical value may be used to specify if parallel computing should be used (TRUE) or not (FALSE, default) for evaluating the fitness function. A numeric value can also be supplied, in which case it gives the number of cores/processors to employ; by default, all the available cores, as provided by detectCores(), are used.

Two types of parallel functionalities are available depending on system OS: on Windows only snow type functionality is present, whereas on POSIX operating systems, such as Unix, GNU/Linux, and Mac OSX, both snow and multicore (default) functionalities are available. In the latter case, a string can be used as the argument to parallel to set out which parallelisation tool should be used.

A final option is available if a researcher plans to use a cluster of multiple machines. In this case, ga() can be executed in parallel using all, or a subset of, the cores available to each machine assigned to the cluster. However, this option requires more work from the user, who needs to set up and register a parallel back end. The resulting cluster object should then be passed as input for the parallel argument.

\section{Islands parallel implementation}

The ISLPGA approach to parallel computing in GA has been implemented in the gaisl() function. This function accepts the same input arguments as the ga() function (see Scrucca, 2013, Section 3), with the following additional arguments:

numIslands

migrationRate

migrationInterval
An integer value which specifies the number of islands to use in the genetic evolution (by default is set to 4 ).

A value in the range $(0,1)$ which gives the proportion of individuals that undergo migration between islands in every exchange (by default equal to $0.10)$.

An integer value specifying the number of iterations at which exchange of individuals takes place. This interval between migrations is called an epoch, and it is set at 10 by default.

The implemented ISLPGA uses a simple ring topology, in which each island is connected unidirectionally with another island, hence forming a single continuous pathway (see Figure 7). Thus, at each exchange step the top individuals, selected according to the specified migrationRate, substitute random individuals (with the exception of the elitist ones) in the connected island.

By default, the function gaisl() uses parallel = TRUE, i.e. the islands algorithm is run in parallel, but other values can also be provided as described in the previous subsection. Note that it is possible to specify a number of islands larger than the number of available cores. In such a case, the parallel 
algorithm will be run using blocks of islands, with the block size depending on the maximal number of cores available or the number of processors as specified by the user.

It has been noted that using parallel islands GAs often leads to, not only faster algorithms, but also superior numerical performance even when the algorithms run on a single processor. This because each island can search in very different regions of the whole search space, thus enhancing the exploratory attitude of evolutionary algorithms.

\section{Simulation study}

In this Section results from a simulation study are presented and discussed. The main goal is to compare the performance of sequential GAs with the two forms of parallel algorithms implemented in the GA package, namely GPGA and ISLPGA, for varying number of cores and different fitness computing times. A fictitious fitness function is used to allow for controlling the computing time required at each evaluation. This is achieved by including the argument pause which suspend the execution for a specified time interval (in seconds):

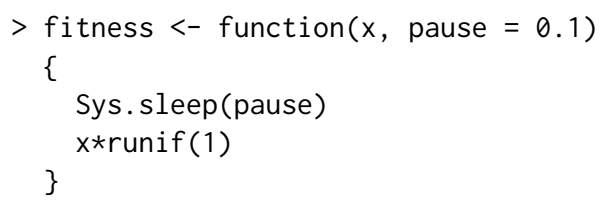

The simulation design parameters used are the following:

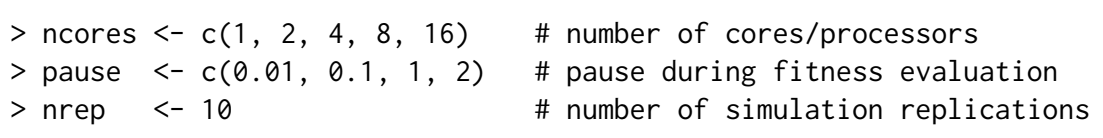

Thus, ncores specifies that up to 16 cores or CPU processors are used in the parallel GAs solutions for increasing time spent on fitness evaluation as specified by pause (in seconds). Each combination of design parameters is replicated $n r e p=10$ times and results are then averaged.

GAs are run under the GPGA approach using popsize $=50$ and maxiter $=100$. For ISLPGA runs the numIslands argument is set at the specified number of cores, with popSize $=160$ and maxiter $=$ 100. The increased population size allows to work with at least 10 individuals on each island when numIslands is set at the maximum number of cores. In both cases, the remaining arguments in ga() or gaisl() function are set at their defaults. Note that run times cannot be compared between the two approaches because they use different population sizes.

The study was performed on a 16 cores Intel ${ }^{\circledR}$ Xeon ${ }^{\circledR}$ CPU E5-2630 running at $2.40 \mathrm{GHz}$ and with 128GB of RAM. The R code used in the simulation study is provided in the accompanying supplemental material.

Graphs in the left panel of Figures 8 and 9 show the average execution times needed for varying number of cores and different fitness computing times. As expected, increasing the number of cores allows to run GAs faster, but the improvement is not linear, in particular for the GPGA approach.

By using a machine with $P$ cores/processors, we would like to obtain an increase in calculation speed of $P$ times. However, this is typically not the case because in the implementation of a parallel algorithm there are some inherent non-parallelisable parts and communication costs between tasks (Nakano, 2012). The speedup achieved using $P$ processors is computed as $s_{P}=t_{1} / t_{P}$, where $t_{i}$ is the execution time spent using $i$ cores. Graphs in the right panel of Figures 8 and 9 show the speedup obtained in our simulation study. For the GPGA approach the speedup is quite good but it is always sub-linear, in particular for the less demanding fitness evaluation time and when the number of cores increases. On the other hand, the ISLPGA implementation shows a very good speedup (nearly linear).

Amdahl's law (Amdahl, 1967) is often used in parallel computing to predict the theoretical maximum speedup when using multiple processors. According to this, if $f$ is the fraction of nonparallelisable task, i.e. the part of the algorithm that is strictly serial, and $P$ is the number of processors in use, then the speedup obtained on a parallel computing platform follows the equation

$$
S_{P}=\frac{1}{f+(1-f) / P} .
$$

In the limit, the above ratio converges to $S_{\max }=1 / f$, which represents the maximum speedup attainable in theory, i.e. by a machine with an infinite number of processors. Figures 10 and 11 show the observed speedup factors $S_{P}$ and the estimated Amdahl's law curves fitted by nonlinear least squares. In all the cases, Amdahl's law appears to well approximate the observed behaviour. The horizontal dashed lines are drawn at the maximum speedup $S_{\max }$, which is computed based on the 

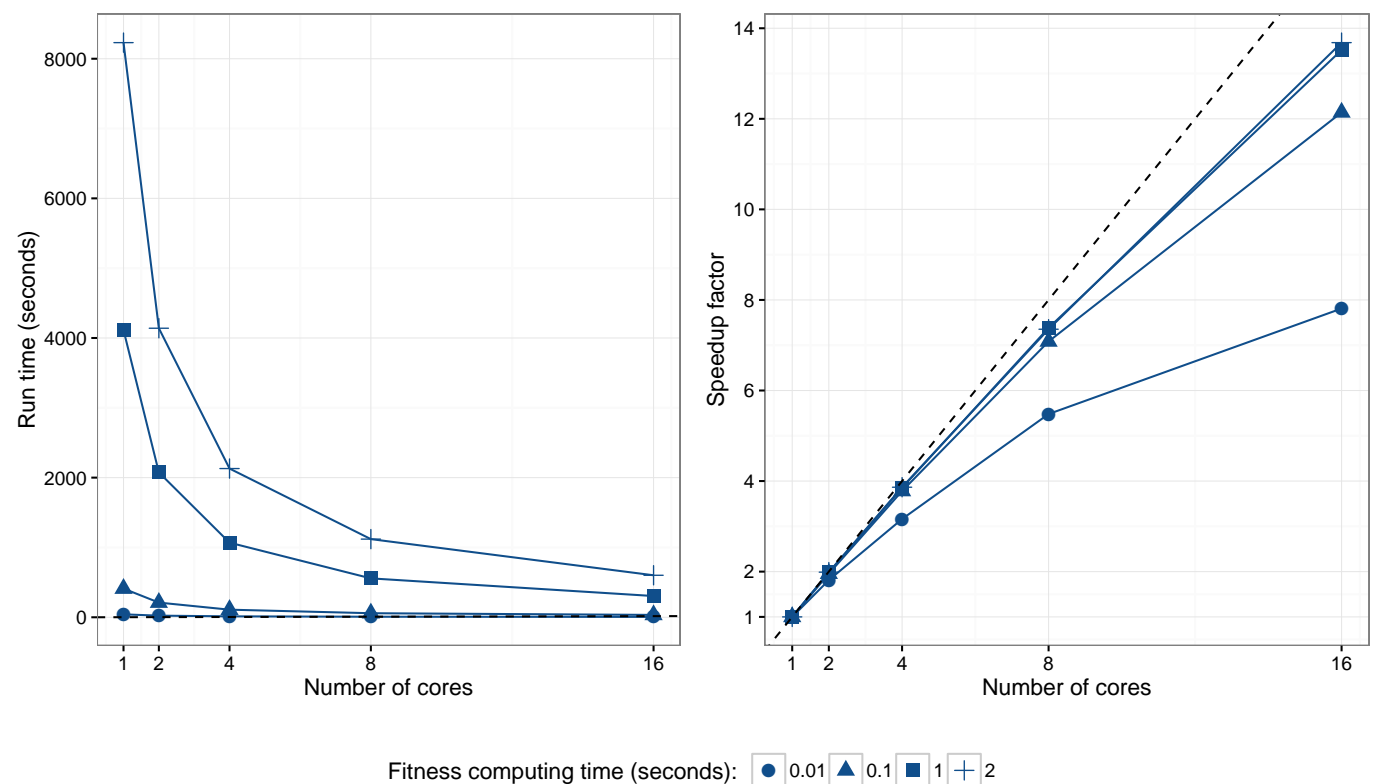

Figure 8: Empirical GPGA performance for varying number of cores/processors and different fitness computing times. Graph on the left panel shows the average running times, whereas graph on the right panel shows the speedup factor compared to the sequential run (i.e. when only 1 core is used). In the latter plot, the dashed line represents the "ideal" linear speedup.
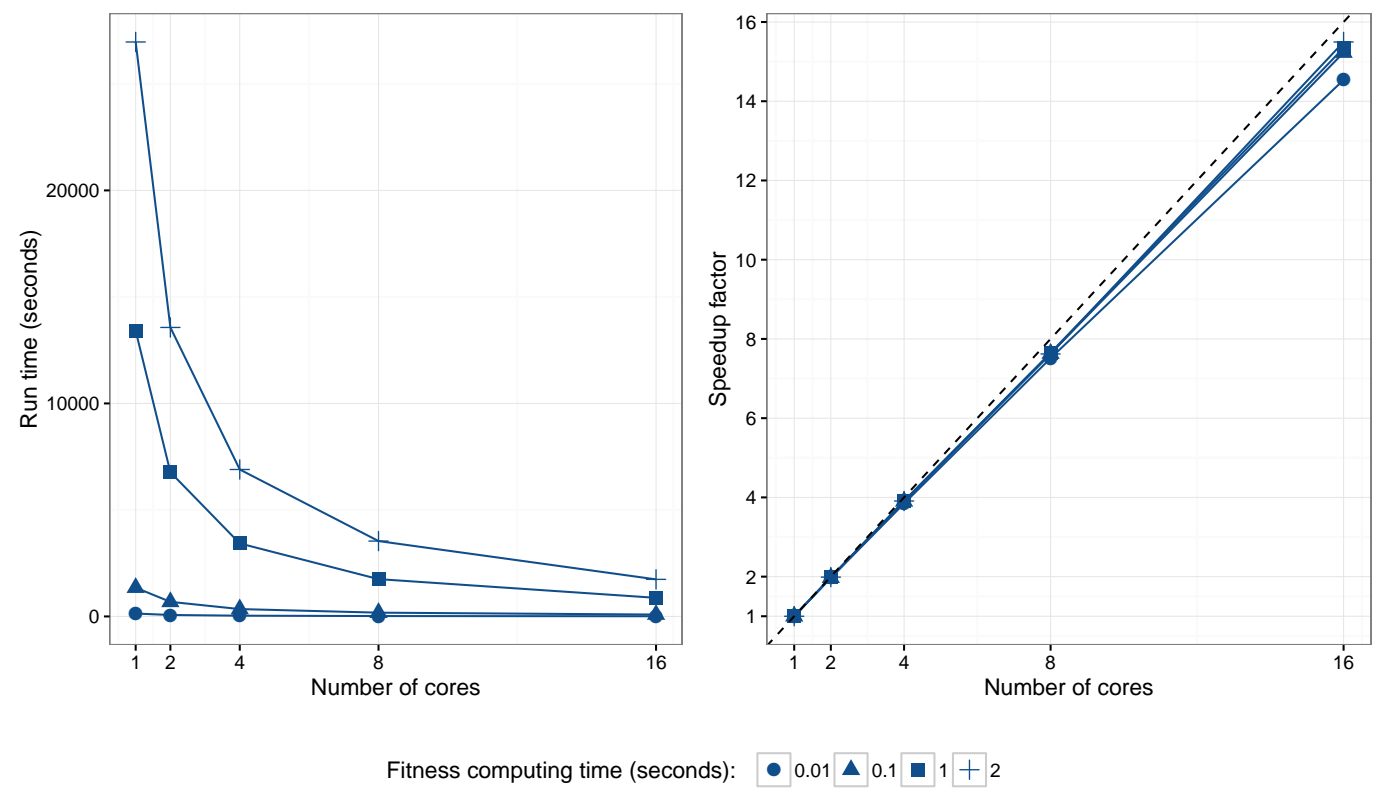

Figure 9: Empirical ISLPGA performance for varying number of cores/processors and different fitness computing times. Graph on the left panel shows the average running times, whereas graph on the right panel shows the speedup factor compared to the sequential run (i.e. when only 1 core is used). In the latter plot, the dashed line represents the "ideal" linear speedup.

estimated fraction of non-parallelisable task $f$ (see also Table 2). As the time required for evaluating the fitness function increases, the maximum speedup attainable also increases. As noted earlier, the ISLPGA approach shows an improved efficiency compared to the simple GPGA. 
Table 2: Fraction of non-parallelisable task $(f)$ estimated by nonlinear least squares using the Amdahl's law (3), and corresponding theoretical speedup $\left(S_{\max }\right)$ for the GPGA and ISLPGA approaches.

\begin{tabular}{|c|c|c|c|c|c|c|c|c|}
\hline \multirow[b]{3}{*}{$f$} & \multicolumn{4}{|c|}{ GPGA } & \multicolumn{4}{|c|}{ ISLPGA } \\
\hline & 0.01 & 0.1 & 1 & 2 & 0.01 & 0.1 & 1 & 2 \\
\hline & 0.0695 & 0.0209 & 0.0122 & 0.0114 & 0.0069 & 0.0036 & 0.0031 & 0.0025 \\
\hline$S_{\max }$ & 14.38 & 47.76 & 81.88 & 87.88 & 145.29 & 278.57 & 327.12 & 408.58 \\
\hline
\end{tabular}

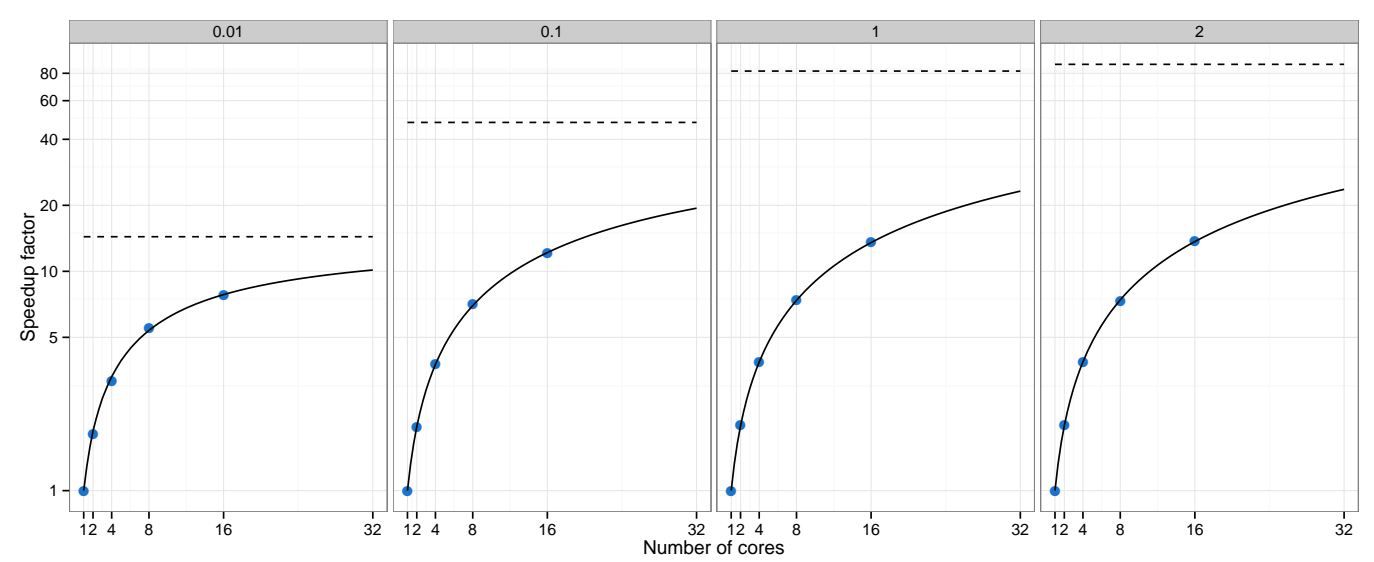

Figure 10: Amdahl's law curves for the GPGA approach. Points refer to the speedup factors observed using different number of cores/processors, whereas the curves are estimated using nonlinear least squares. Horizontal dashed lines refer to the maximum speedup theoretically attainable. Each panel corresponds to a different fitness computing time (in seconds), and vertical axes are on log scale.

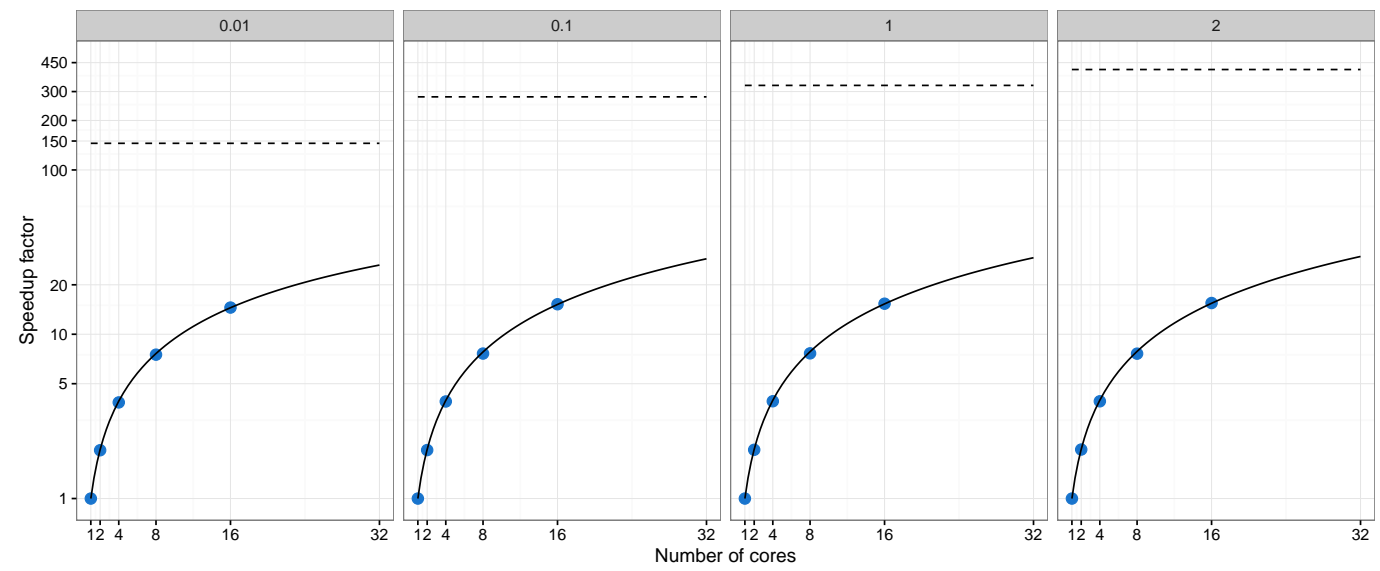

Figure 11: Amdahl's law curves for the ISLPGA approach. Points refer to the speedup factors observed using different number of cores/processors, whereas the curves are estimated using nonlinear least squares. Horizontal dashed lines refer to the maximum speedup theoretically attainable. Each panel corresponds to a different fitness computing time (in seconds), and vertical axes are on log scale.

\section{ARIMA order selection}

Autoregressive moving average (ARMA) models are a broad class of parametric models for stationary time series popularised by Box and Jenkins (1976). They provide a parsimonious description of a stationary stochastic process in terms of two polynomials, one for the auto-regression and the second for the moving average. Nonstationay time series can be modelled by including an initial differencing step ("integrated" part of the model). This leads to autoregressive integrated moving average (ARIMA) models, a popular modelling approach in real-world processes.

ARIMA models can be fitted by MLE after identifying the order $(p, d, q)$ for the autoregressive, integrated, and moving average components, respectively. This is typically achieved by preliminary inspection of the autocovariance function (ACF) and partial autocovariance function (PACF). Model selection criteria, such as the Akaike information criterion (AIC), the corrected AIC (AICc), and the 
Bayesian information criterion (BIC), are also used for order selection.

The function auto.arima() in package forecast (Hyndman, 2016; Hyndman and Khandakar, 2008) provides an automatic algorithm which combines unit root tests, minimisation of the AICc in a stepwise greedy search, and MLE, to select the order of an ARIMA model. Here, an island parallel GAs approach is used for order selection.

Consider the quarterly U.S. GNP from 1947(1) to 2002(3) expressed in billions of chained 1996 dollars and seasonally adjusted. The data are available on package astsa and described in Shumway and Stoffer (2013).

$>\operatorname{data}($ gnp, package="astsa")

$>\operatorname{plot}(g n p)$

The plot of the time series obtained with the last command is shown in Figure 12a.

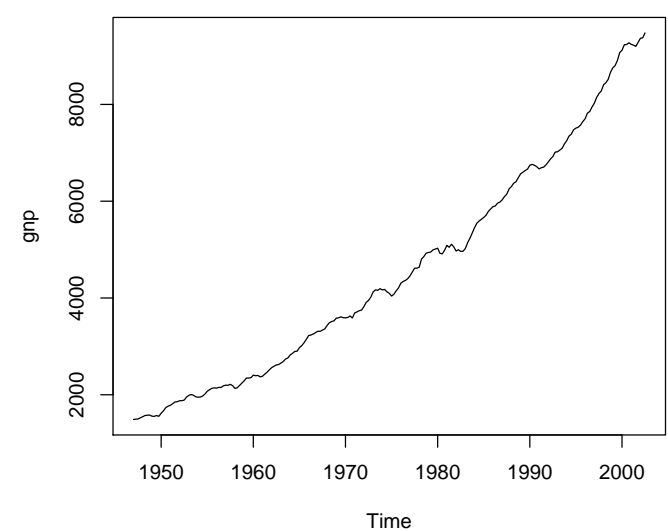

(a)

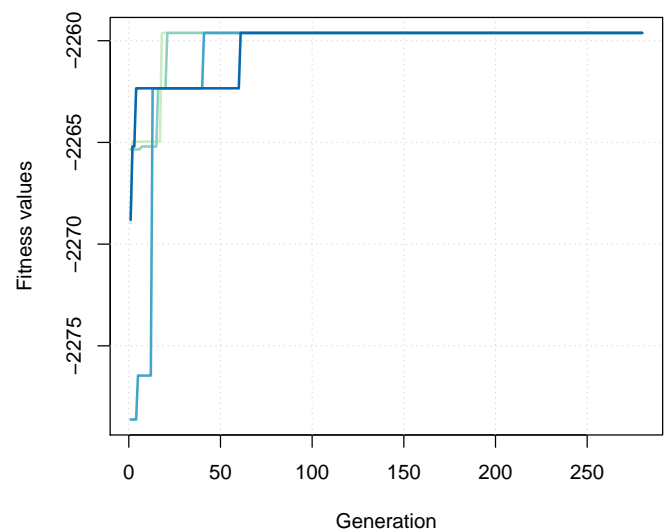

(b)

Figure 12: (a) Plot of quarterly U.S. GNP from 1947(1) to 2002(3). (b) Trace of island parallel GAs search for ARIMA order selection.

The selection of the "optimal" $\operatorname{ARIMA}(p, d, q)$ model can be pursued by using binary GAs to maximise the BIC. The decision variables to be optimised are expressed in binary digits using the following function:

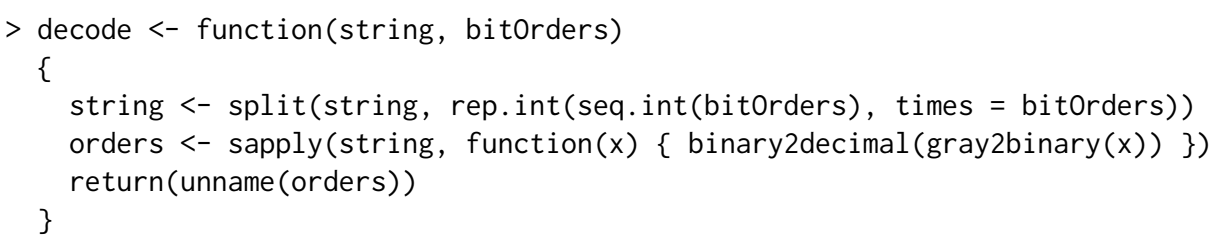

For example, using 3 bits for encoding $p$ and $q$, and 2 bits for $d$, an ARIMA $(3,1,1)$ model can be expressed with the binary string $(0,1,0,0,1,0,0,1)$ :

$>\operatorname{decode}(c(0,1,0,0,1,0,0,1)$, bitOrders $=c(3,2,3))$

[1] 311

Note that the decode() function assumes that the input binary string is expressed using Gray encoding, which ensures that consecutive values have the same Hamming distance (Hamming, 1950).

The fitness function to be used in the GA search is defined as follows:

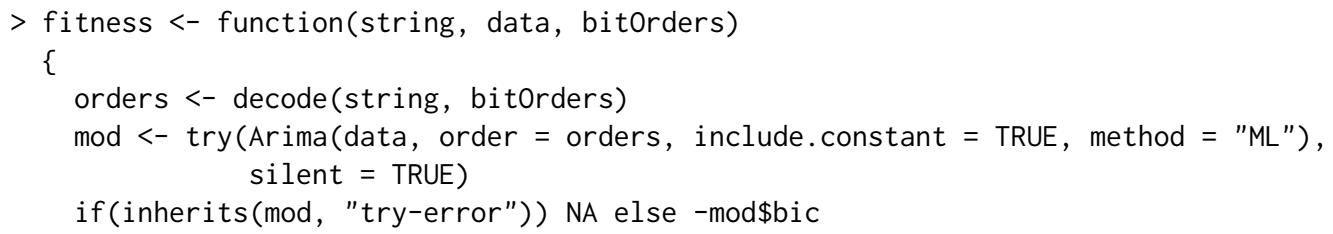

Note that the objective function is defined as (minus) the BIC for the specified ARIMA model, with the latter fitted using the Arima() function available in the $\mathrm{R}$ package forecast. If a different criterion 
for model selection is preferred, for instance the Akaike information criterion (AIC), only the last line of the above fitness() function needs to be modified.

An island binary parallel GA is then used to search for the best ARIMA model, using a migration interval of 20 generations, and the default migration rate of 0.1 :

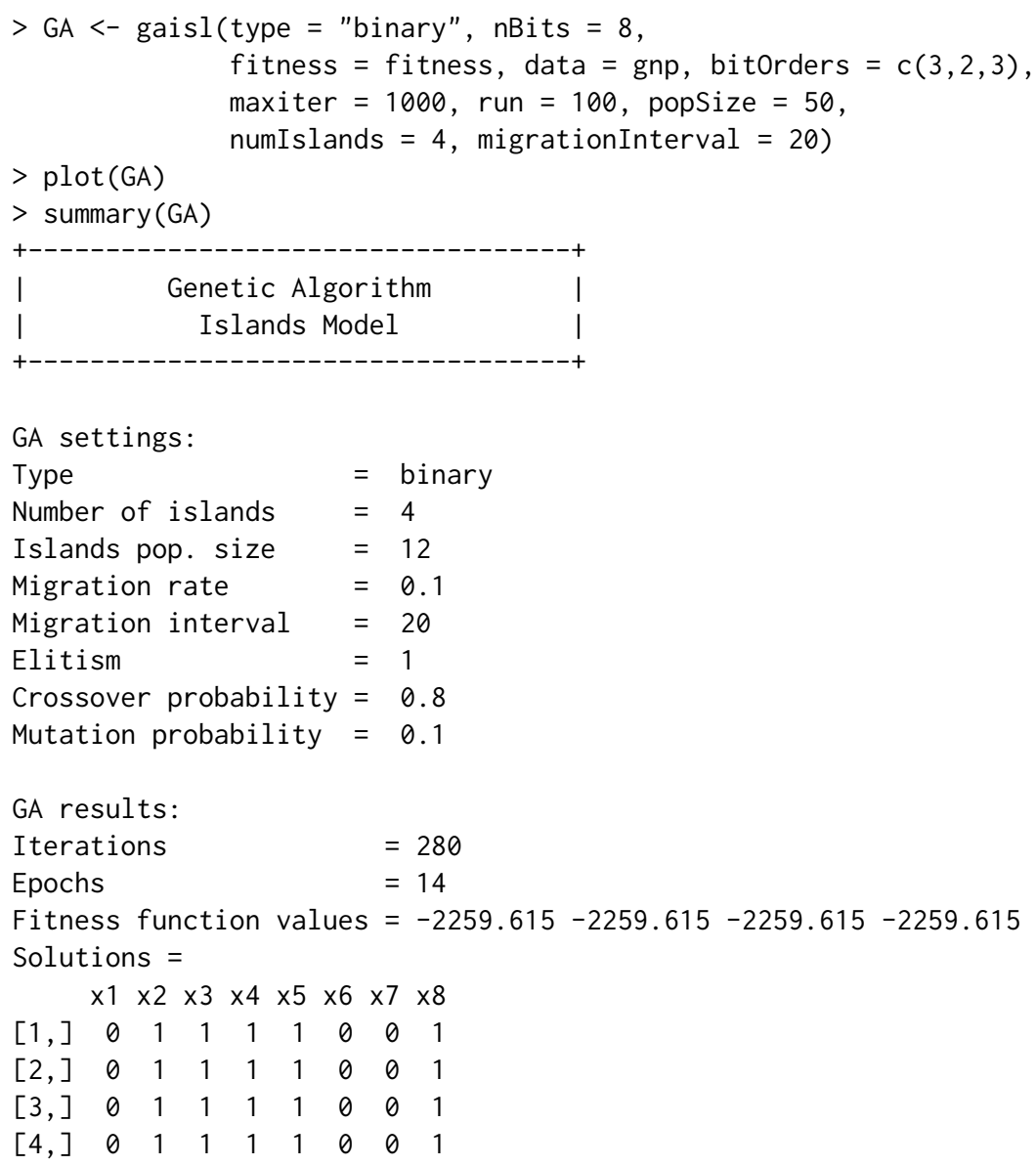

Figure $12 \mathrm{~b}$ shows the trace of the ISLPGA search for each of the four islands used. All the islands converge to the same final solution, as also shown by the summary output above. The selected model is an $\operatorname{ARIMA}(2,2,1)$, which can be fitted using:

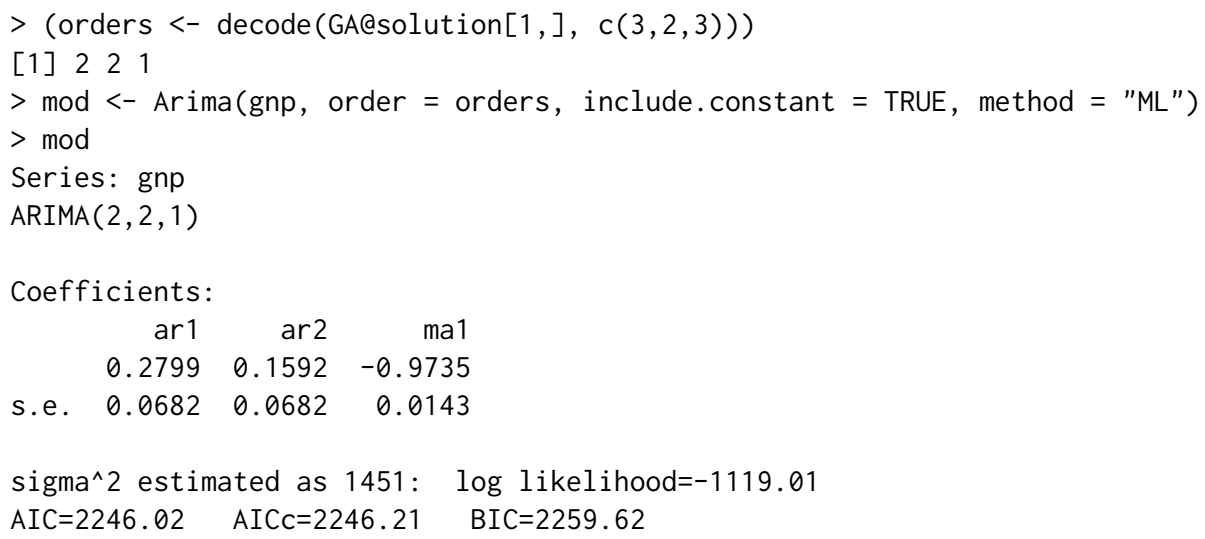

It is interesting to compare the above solution with that obtained with the automatic procedure implemented in auto.arima() using the same criterion:

$>\bmod 1<-$ auto.arima (gnp, ic $=$ "bic")

$>\operatorname{print}(\bmod 1)$

Series: gnp

$\operatorname{ARIMA}(1,2,1)$

Coefficients: ar1 ma1 


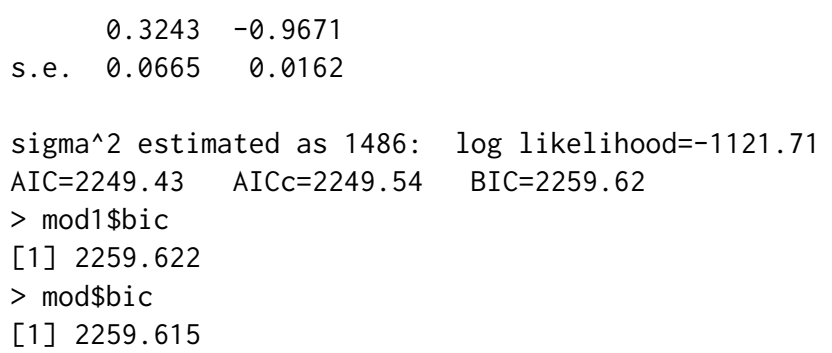

The model returned by auto. arima() is an ARIMA $(1,2,1)$, so a simpler model where an AR(1) component is chosen instead of an AR(2). The BIC values are almost equivalent, with a slightly smaller value for the ARIMA $(2,2,1)$ model identified by ISLPGA. However, by looking at some diagnostic plots it seems that a second-order AR component is really needed to account for autocorrelation at several lags as indicated by the Ljung-Box test of autocorrelation (see Figure 13; the code used to produce the plots is available in the supplementary material).
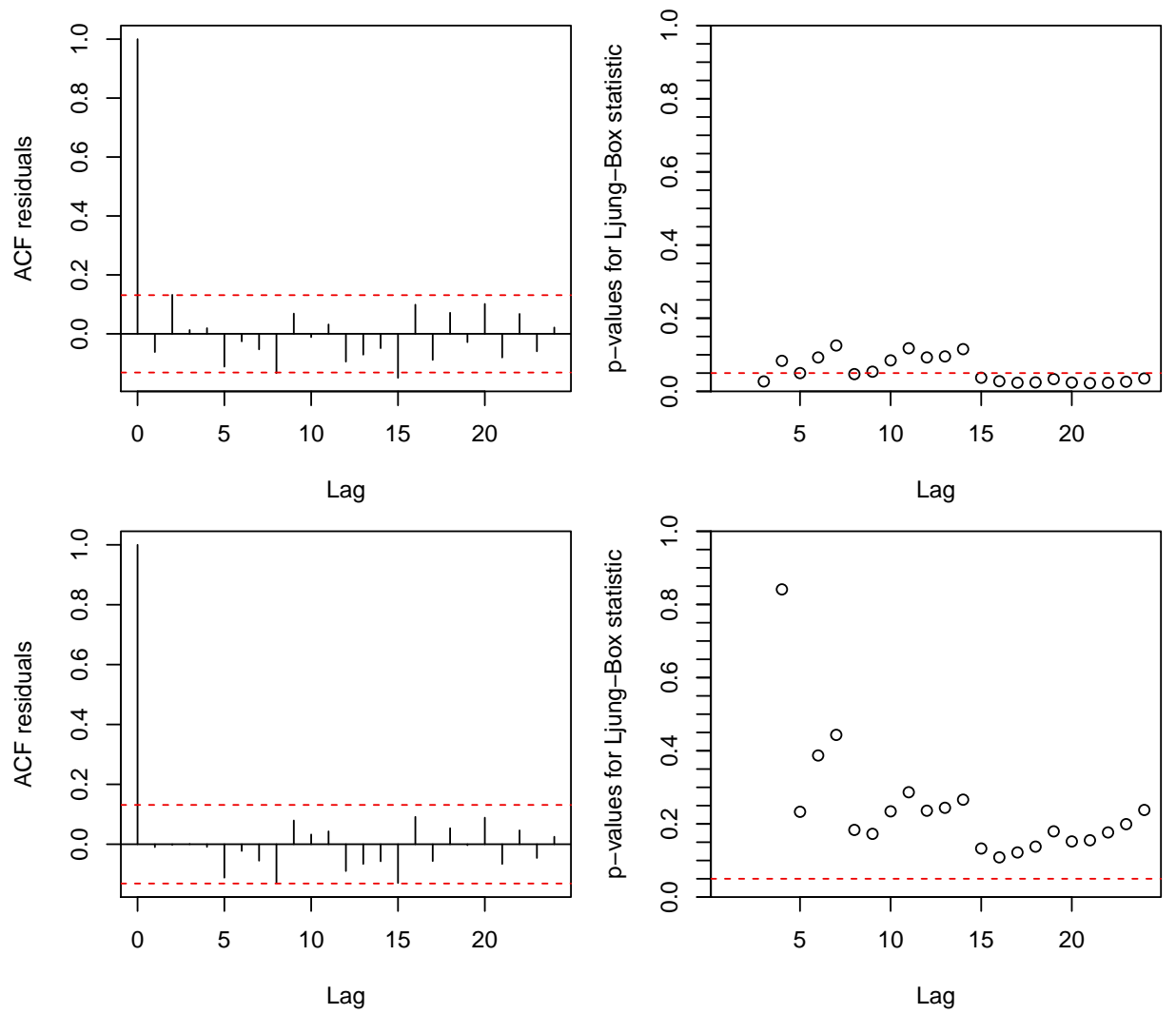

Figure 13: ACF of residuals and p-values for the Ljung-Box test of autocorrelation for the ARIMA $(1,2,1)$ model (top graphs) and the ARIMA $(2,2,1)$ model (bottom graphs) fitted to the quarterly U.S. GNP data from 1947(1) to 2002(3).

\section{Benchmark function optimisation}

Mullen (2014) compared several optimisation algorithms using 48 benchmark functions available in the globalOptTests package. GA was one of the several R packages investigated in such a comparison. However, with the settings used in this study, its overall performance was not particularly brilliant, ranking 14th out of 18 methods, thus leaving plenty of room for improvements. For instance, the Griewank function was one of the most problematic cases, and it is defined as

$$
f\left(x_{1}, \ldots, x_{d}\right)=1+\frac{1}{4000} \sum_{i=1}^{d} x_{i}^{2}-\prod_{i=1}^{d} \cos \left(x_{i} / \sqrt{i}\right) .
$$

This a multimodal, non-separable function, with several local optima within the search region. For any dimensionality $d$, it has one global minimum of zero located at the point $(0, \ldots, 0)$. 
We replicated the simulation study in Mullen (2014) using the standard sequential GA (GA), the parallel island GA with 4 islands (GAISL), the hybrid GA with local search (HGA), and the island GA with local search (HGAISL). Results for the Griewank function based on 100 replications are shown in Figure 14. The use of hybrid GAs, particularly in combination with the islands evolution, clearly yields more accurate solutions and with less dispersion. The same behavior has been observed in many other benchmark functions available in the globalOptTests package.

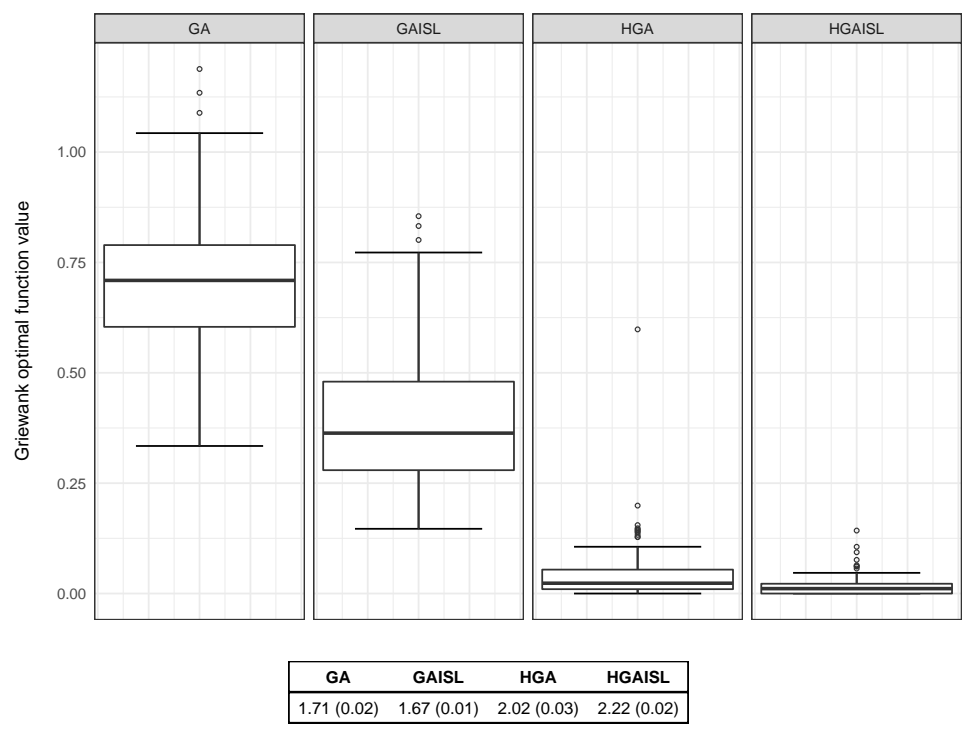

Figure 14: Results from 100 replications of Griewank function optimisation using standard GAs (GA), island GAs (GAISL), hybrid GAs with local search (HGA), and island GAs with local search (HGAISL). The table at the bottom reports the average (and standard deviation) computing times in seconds used by each algorithm.

\section{Summary}

GA is a flexible R package for solving optimisation problems with genetic algorithms. This paper presents some improvements recenquadrtly added to the package. We have discussed the implementation of hybrid GAs, which employ local searches during the evolution of a GA to improve accuracy and efficiency. Further speedup can also be achieved by parallel computing. This has been implemented following two different approaches. In the first one, the so-called master-slave approach, the fitness function is evaluated in parallel, either on a single multi-core machine or on a cluster of multiple computers. In the second approach, called islands model, the evolution takes place independently on several sub-populations assigned to different islands, with occasional migration of solutions between islands. Both enhancements often lead to high-quality solutions more efficiently.

Future plans include the possibility to improve the overall performance by rewriting some key functions in $\mathrm{C}++$ using the Rcpp package. In particular, coding of genetic operators in $\mathrm{C}++$ should provide sensible benefits in terms of computational speedup. Another interesting option to perform local search is the inclusion of Hooke-Jeeves direct search algorithm as described in Satman (2015). Finally, the package memoise enables to store the results of an expensive fitness function call and returns the cached result when the same input arguments occur again. This strategy could be conveniently employed in the case of binary and permutation GAs.

\section{Acknowledgements}

The author acknowledge the CINECA award under the ISCRA initiative (http://www. hpc. cineca. it/services/iscra) for the availability of high performance computing resources and support.

\section{Bibliography}

G. M. Amdahl. Validity of the single processor approach to achieving large scale computing capabilities. In AFIPS Conference Proceedings, volume 30, pages 483-485, 1967. [p198] 
T. Back, D. B. Fogel, and Z. Michalewicz. Evolutionary Computation 1: Basic Algorithms and Operators. IOP Publishing Ltd., Bristol and Philadelphia, 2000a. [p187]

T. Back, D. B. Fogel, and Z. Michalewicz. Evolutionary Computation 2: Advanced Algorithms and Operators. IOP Publishing Ltd., Bristol and Philadelphia, 2000b. [p187]

G. E. Box and G. M. Jenkins. Time Series Analysis: Forecasting and Control. Holden-Day, San Francisco, CA, 1976. [p200]

B. P. Carlin, A. E. Gelfand, and A. F. Smith. Hierarchical bayesian analysis of changepoint problems. Applied Statistics, 41:389-405, 1992. [p193, 195]

E. K. Chong and S. H. Zak. An Introduction to Optimization. John Wiley \& Sons, Hoboken, New Jersey, 2013. [p187]

P. Cortez. Modern Optimization with R. Springer-Verlag, 2014. [p187]

K. A. De Jong. Evolutionary Computation: a Unified Approach. MIT press, 2006. [p187]

M. Dorigo and T. Stützle. Ant Colony Optimization. The MIT Press, Cambridge, 2004. [p187]

D. Eddelbuettel. CRAN task view: High-performance and parallel computing with R, 2016. URL http: //CRAN.R-project.org/view=HighPerformanceComputing. Version 2016-01-05. [p196]

A. E. Eiben and C. A. Schippers. On evolutionary exploration and exploitation. Fundamenta Informaticae, 35:35-50, 1998. [p188]

A. E. Eiben and J. E. Smith. Introduction to Evolutionary Computing. Springer-Verlag, Berlin Heidelberg, 2003. [p187, 188]

M. Gilli and E. Schumann. Heuristic optimisation in financial modelling. Annals of Operations Research, 193(1):129-158, 2012. [p190]

M. Gilli, D. Maringer, and E. Schumann. Numerical Methods and Optimization in Finance. Academic Press, 2011. [p189]

G. H. Givens and J. A. Hoeting. Computational Statistics. John Wiley \& Sons, 2nd edition edition, 2013. [p187]

F. Glover and M. Laguna. Tabu Search. Springer-Verlag, 2013. [p187]

R. W. Hamming. Error detecting and error correcting codes. Bell System technical journal, 29(2):147-160, 1950. [p201]

N. Hansen. The cma evolution strategy: a comparing review. In J. A. Lozano, P. Larrañga, I. Inza, and E. Bengoetxea, editors, Towards a New Evolutionary Computation. Advances in Estimation of Distribution Algorithms., pages 75-102. Springer-Verlag, 2006. [p187]

R. J. Hyndman. forecast: Forecasting Functions for Time Series and Linear Models, 2016. URL http: //github.com/robjhyndman/forecast. R package version 7.3. [p201]

R. J. Hyndman and Y. Khandakar. Automatic time series forecasting: The forecast package for R. Journal of Statistical Software, 26(3):1-22, 2008. URL http://www. jstatsoft. org/article/view/v027i03. [p201]

R. G. Jarrett. A note on the intervals between coal-mining disasters. Biometrika, 66(1):191-193, 1979. [p193]

J. Kennedy and R. Eberhart. Particle swarm optimization. In Neural Networks, 1995. Proceedings., IEEE International Conference on, volume 4, pages 1942-1948, 1995. URL https://doi . org/10.1109/icnn. 1995. 488968. [p187]

S. Kirkpatrick, C. D. Gelatt, and M. P. Vecchi. Optimization by simulated annealing. Science, 220(4598): 671-680, 1983. [p187]

J. K. Lindsey. Statistical Analysis of Stochastic Processes in Time. Cambridge University Press, 2004. [p192]

H. R. Lourenço, O. C. Martin, and T. Stützle. Iterated Local Search. Springer-Verlag, 2003. [p187]

S. Luke. Essentials of Metaheuristics. Lulu, 2nd edition, 2013. URL http://cs.gmu. edu/ sean/book/ metaheuristics. Freely available at http://cs.gmu.edu/ sean/book/metaheuristics/. [p187] 
G. Luque and E. Alba. Parallel Genetic Algorithms: Theory and Real World Applications. Springer-Verlag, 2011. [p196]

E. McCallum and S. Weston. Parallel R. O’Reilly Media, 2011. [p196]

K. M. Mullen. Continuous global optimization in R. Journal of Statistical Software, 60(6):1-45, 2014. URL http: //www. jstatsoft.org/v60/i06/. [p203, 204]

J. Nakano. Parallel computing techniques. In J. E. Gentle, W. K. Härdle, and Y. Mori, editors, Handbook of Computational Statistics, pages 243-271. Springer-Verlag, 2nd ed. edition, 2012. [p198]

J. C. Nash. Nonlinear Parameter Optimization Using R Tools. John Wiley \& Sons, 2014. [p187]

C. H. Papadimitriou and K. Steiglitz. Combinatorial Optimization: Algorithms and Complexity. Dover Publications, Inc., Mineola, NY, 1998. [p187]

A. Raftery and V. Akman. Bayesian analysis of a poisson process with a change-point. Biometrika, 73 (1):85-89, 1986. [p193, 195]

D. Ruppert and D. S. Matteson. Statistics and Data Analysis for Financial Engineering. Springer-Verlag, 2nd edition, 2015. URL https://doi .org/10.1007/978-1-4939-2614-5. [p189]

M. H. Satman. Hybridization of floating-point genetic algorithms using Hooke-Jeeves algorithm as an intelligent mutation operator. Journal of Mathematical and Computational Science, 5(3):320, 2015. [p204]

G. Schwartz. Estimating the dimension of a model. The Annals of Statistics, 6:31-38, 1978. [p195]

L. Scrucca. GA: A package for genetic algorithms in R. Journal of Statistical Software, 53(4):1-37, 2013. URL http: //www. jstatsoft.org/v53/i04/. [p188, 197]

R. H. Shumway and D. S. Stoffer. Time Series Analysis and Its Applications. Springer-Verlag, 3rd edition, 2013. [p201]

D. Simon. Evolutionary Optimization Algorithms. John Wiley \& Sons, 2013. [p187]

R. Storn and K. Price. Differential evolution - a simple and efficient heuristic for global optimization over continuous spaces. Journal of Global Optimization, 11(4):341-359, 1997. ISSN 1573-2916. URL https://doi.org/10.1023/a:1008202821328. [p187]

S. Theussl and H. W. Borchers. CRAN task view: Optimization and mathematical programming, 2015. URL http://CRAN.R-project.org/view=0ptimization. Version 2015-11-27. [p187]

M. Črepinšek, S.-H. Liu, and M. Mernik. Exploration and exploitation in evolutionary algorithms: A survey. ACM Comput. Surv., 45(3):1-35, 2013. ISSN 0360-0300. URL https: //doi .org/10.1145/ 2480741.2480752. [p188]

D. Whitley. A genetic algorithm tutorial. Statistics and Computing, 4(2):65-85, 1994. [p188, 196]

Luca Scrucca

Università degli Studi di Perugia

Via A. Pascoli 20, 06123 Perugia

Italy

luca.scrucca@unipg. it 